\title{
Fault diagnosis and condition monitoring of wind turbines
}

Niemann, Hans Henrik; Poulsen, Niels Kjølstad; Mirzaei, Mahmood; Henriksen, Lars Christian

Published in:

International Journal of Adaptive Control and Signal Processing

Link to article, DOI:

10.1002/acs.2782

Publication date:

2017

Document Version

Peer reviewed version

Link back to DTU Orbit

Citation (APA):

Niemann, H. H., Poulsen, N. K., Mirzaei, M., \& Henriksen, L. C. (2017). Fault diagnosis and condition monitoring of wind turbines. International Journal of Adaptive Control and Signal Processing, 32(4), 586-613.

https://doi.org/10.1002/acs.2782

\section{General rights}

Copyright and moral rights for the publications made accessible in the public portal are retained by the authors and/or other copyright owners and it is a condition of accessing publications that users recognise and abide by the legal requirements associated with these rights.

- Users may download and print one copy of any publication from the public portal for the purpose of private study or research.

- You may not further distribute the material or use it for any profit-making activity or commercial gain

- You may freely distribute the URL identifying the publication in the public portal

If you believe that this document breaches copyright please contact us providing details, and we will remove access to the work immediately and investigate your claim. 


\title{
Fault diagnosis and condition monitoring of wind turbines
}

\author{
Henrik Niemann ${ }^{1}{ }^{10}$ | Niels Kjølstad Poulsen ${ }^{2}$ | Mahmood Mirzaei ${ }^{3}$ | Lars Christian Henriksen ${ }^{4}$
}

\author{
${ }^{1}$ Department of Electrical Engineering, \\ Automation and Control, Technical \\ University of Denmark, Kgs. Lyngby, \\ Denmark \\ ${ }^{2}$ Department of Applied Mathematics and \\ Computer Science, Technical University of \\ Denmark, Kgs. Lyngby, Denmark \\ ${ }^{3}$ Department of Wind Energy, Technical \\ University of Denmark, Roskilde, Denmark \\ ${ }^{4}$ AF Consult, Lyskær 3F Herlev, Denmark

\section{Correspondence} \\ Henrik Niemann, Department of Electrical \\ Engineering, Automation and Control, \\ Build. 326, Technical University of \\ Denmark, DK-2800 Kgs. Lyngby, Denmark. \\ Email: hhn@elektro.dtu.dk
}

\begin{abstract}
Summary
This paper describes a model-free method for the fault diagnosis and condition monitoring of rotor systems in wind turbines. Both fault diagnosis and monitoring can be achieved without using a model for the wind turbine, applied controller, or wind profiles. The method is based on measurements from standard sensors on modern wind turbines, including moment sensors and rotor angle sensors. This approach will allow the method to be applied to existing wind turbines without any modifications. The method is based on the detection of asymmetries in the rotor system caused by changes or faults in the rotor system. A multiblade coordinate transformation is used directly on the measured flap-wise and edge-wise moments followed by signal modulation. Changes or faults in the rotor system will result in unique signatures in the set of modulation signals. These signatures are described through the amplitudes and phase information of the modulation signals. It is possible to detect and isolate which blade is faulty or has been changed based on these signatures. Furthermore, the faulty component can be isolated, ie, the actuator, sensor or blade, and the type of fault can be determined. The method can be used both on- and off-line.
\end{abstract}

\section{KEYWORDS}

blade faults, model-free fault diagnosis, multiblade coordinate transformation, system monitoring, signal modulation, wind turbines

\section{1 | INTRODUCTION}

This paper describes a method for the fault diagnosis (detection and isolation) and condition monitoring of a wind turbine rotor. Fault diagnosis and condition monitoring of wind turbines is important during breakdowns of wind turbines as well as loss of power production in the case of faults and changes in the wind turbine. Thus, it is crucial to be able to monitor, detect, and isolate changes and faults before they become critical and have a significant impact on the integrity of the wind turbine, with a resultant reduction in power production. In more critical cases, the wind turbine might also be damaged because faults and changes. Thus, it is also important to be able to monitor critical elements to prevent a reduction in power production and reduce the risk of damage to the turbine. Such monitoring is particularly important for offshore wind turbines, which are difficult and expensive to maintain.

\subsection{Survey of existing results}

In general, 2 types of methods exist for fault diagnosis and monitoring: model-based and model-free methods. The latter type of methods can also be called data-driven methods. Both types of methods have been applied to wind turbines.

In model-based methods, as described in, eg, previous studies, ${ }^{1-3}$ real measurements of the real system are compared with a model of the system. One method for doing this comparison is by using observers or Kalman filters. Residual vectors 
are generated as the difference between the real output from the system and the estimated output from the observers or Kalman filters. A fault will change the output from the system and will result in a change in the residual output. This change can be used to detect, isolate, and estimate the fault. Statistical test methods, eg, CUSUM or GLR tests, ${ }^{4,5}$ can also be applied for detection and isolation. Using statistical test methods for residual signals results in a reliable detection method that enables both the detection time and the time between false alarms (wrong detection) to be guaranteed. However, model-based methods have the drawback that they require a reasonably good model to be able to guarantee the detection and isolation of faults.

An alternative to model-based methods is data-driven or model-free methods, where fault detection and isolation are based on different types of data and signal analysis (see, eg, $\operatorname{Qin}^{6}$ ). Model-free methods can be based on either frequency domain approaches or time domain approaches. Diagnosis can be based on the detection of changes in mean values, variances, a change in trends, signature analysis, wavelet analysis, or neural networks, among many different methods. These methods are similar in that they are not directly based on a detailed model of the system. Instead, these types of methods require a "normal picture" of the system to compare the real system with the "normal picture." The quality of the fault diagnosis depends directly upon how well this normal picture is described.

Finally, monitoring and diagnosis can also be based on dedicated sensors in central mechanical elements. It is possible, for example, to include a temperature sensor in a gearbox to monitor the internal temperature. Data from these types of sensors typically require on-line monitoring or on-line data analysis.

Fault diagnosis of wind turbines has been considered in many publications in recent years. The central papers in this topic include: Hameed et al, ${ }^{7}$ Marquez et al,${ }^{8}$ Tchakoua et al,${ }^{9}$ Sheng and Veers, ${ }^{10}$ Wei et al, ${ }^{11}$ Wei and Verhaegen, ${ }^{12}$ Saad et al,,${ }^{13}$ Watson et al, ${ }^{14}$ Gong and Qiao, ${ }^{15}$ Feng et al, ${ }^{16}$ Amirat et al,,${ }^{17}$ Qiao, ${ }^{18}$ Hang et al,${ }^{19}$ and Kusiak et al ${ }^{20}$ The first 3 papers, Hameed et al, ${ }^{7}$ Marquez et al, ${ }^{8}$ and Tchakoua et al, ${ }^{9}$ are survey papers considering the condition monitoring of wind turbines. In Tchakoua et al, ${ }^{9}$ future challenges and new trends are discussed. An overview of methods for the condition monitoring of wind turbine drivetrains is given in Sheng and Veers. ${ }^{10}$ In the 2 papers by Wei et al, ${ }^{11,12}$ Kalman filters and robust observers are considered for sensor and actuator fault diagnosis. The fault diagnosis of wind turbine generators is investigated in Saad et al ${ }^{13}$ and Watson et al. ${ }^{14}$ Here, frequency estimation and wavelet methods are used. The wind turbine generator is also considered in Gong and $\mathrm{Qiao}^{15}$ in relation to imbalance faults. The generator frequency is estimated based on measurements of the 1-phase stator current of the generator and imbalances can be detected on the basis of this estimation. Different diagnosis methods based on modulation and demodulation are considered in Feng et al, ${ }^{16}$ Amirat et al, ${ }^{17}$ and Qiao. ${ }^{18}$ In the first 2 papers, fault diagnosis is restricted to gearboxes and generators. In Qiao, ${ }^{18}$ the diagnosis is restricted to not only generators and gearboxes but also faults on other parts of the turbines. Fault diagnosis based on sensor fusion is considered in Hang et al. ${ }^{19}$ Faults can be detected and isolated on the basis of the signals from sensor fusion and individual classifiers. This is a model-free method. In the last paper mentioned above ${ }^{20}$ the concept of data-driven fault diagnosis is applied. Five different algorithms are tested for the fault diagnosis and monitoring of blade pitch faults. Furthermore, a benchmark model for fault diagnosis of wind turbines is developed in Odgaard et $\mathrm{a}^{21}$ to stimulate the research activity in this area. Several different methods have been applied and compared on the basis of this benchmark model. The results of the different diagnosis methods applied to the benchmark model can be found in journals and different conferences from recent years.

An issue related to the fault diagnosis of wind turbines is the applied control strategy. The control of a wind turbine depends on the wind speed. Below the rated wind speed, the wind turbine operates in partial load, where the power production is maximized. The partial load operation is normally attained with constant pitch angles for the blades and with a power reference depending on a variable speed. Above the rated wind speed, the wind turbine is in full load operation and has reached maximal power production and rated speed. In full-load operation, the pitch angles are controlled to obtain a constant speed, and the power production is kept at the rated power. The wind turbine blades can be controlled in 2 ways: collective and individual pitch control. The pitch angles are the same in the first case, whereas the pitch angles are controlled individually in the second case. Individual pitch control is more complex than collective pitch control; however, it is needed in many cases due to the increasing size of the wind turbines, which also results in a variation in the wind field over the rotor disk. Thus, fault diagnosis depends on the operation state of the turbine, namely, whether it is below or above the rated wind speed.

\section{2 | Contribution}

This paper presents a new method for the monitoring and fault diagnosis of wind turbine rotor systems. The method is a model-free diagnosis method that does not require a model of the wind turbine or rotor system. Moreover, descriptions of the wind profile and applied controller are also not needed. 
The central element in the method is the analysis of the pattern of variation in the root bending moments for each blade. These root bending moments are analyzed with respect to any asymmetry caused by faults or changes in the rotor system. Faults or changes in the rotor system that do not result in asymmetric root bending moments cannot be detected by the method described in this paper. The method can be used both on- and off-line.

The suggested method consists of 2 parts:

1. A direct transformation of the measurements of the root bending moments using the multiblade coordinate (MBC) transformation followed by signal modulation on the transformed root moment signals with respect to the rotor speed.

2. A signature analysis of the transformed root bending moments based on signal modulation with respect to isolating faults or changes in the rotor system.

The MBC transformation, also known as the Coleman transformation, has been used in the fault diagnosis of wind turbines. The MBC transformation was first used in Wei et al ${ }^{11}$ and Wei and Verhaegen ${ }^{12}$ and later in Henriksen et al. ${ }^{22-24}$ The methods described in these papers are model-based methods that require a detailed model of the wind turbine system. The method described in Henriksen et $\mathrm{al}^{22-24}$ is similar to the method described in this paper, except that it is based on a detailed model. The results given in these papers can be compared with the simulation results provided below. A detailed description of the MBC transformation is provided in $\mathrm{Bir}^{25}$

The results of the analysis in part 2 are signatures that are unique and depend directly on the faults or changes in the rotor system. This makes it possible to isolate different faults or changes from each other. The signatures are based on both amplitude and phase information from the modulation signals.

Part 1: The applied MBC transformation transforms between a rotating and fixed frame. The 2 sets of flap-wise and edge-wise root bending moments are measured and then transformed using the standard MBC transformation. The resulting MBC-transformed moments are constant for a symmetrical rotor system and include different periodic components in the case of a nonsymmetrical rotor system. It is then reasonably easy to detect and monitor changes or faults in the rotor system. Isolation of these changes or faults is more complex.

Part 2: Different faults or changes will result in different fingerprint signatures in the MBC output signals. These signatures will occur as the presence of $1 p$ (basic rotational frequency) and/or $2 p$ (double rotational frequency) components in the MBC output signals. Signal modulation with respect to $1 p$ and $2 p$ periodic signals are applied to extract these signatures from the MBC output signals. The signal modulation transforms the periodic components in the MBC output signals into constant components. The signatures from the faults in the rotor system are then transformed into constant components in the $1 p$ and $2 p$ modulation signals. It is possible to isolate the different faults or changes in the rotor system using information about the amplitude and phase of these components.

The following types of faults or changes in the rotor system can be detected and isolated based on the phase analysis of the modulation signals in part 3:

- A blade that is faulty.

- A component that is faulty on a specific blade (sensor fault, actuator fault, or an internal blade fault).

- The type of faults (additive or multiplicative).

Further, it is also possible to estimate the fault indirectly. This will be discussed briefly at the end of the paper.

\section{3 | Structure of the paper}

The remainder of this paper is organized as follows. A short description of wind turbines and their control is given in Section 2. The applied MBC transformation and signal modulation are analyzed in detail in Section 3. The model-free fault diagnosis method based on MBC transformation and signal modulation is described in Section 4. Simulation results are presented in Section 5 and discussed in Section 6. The paper closes with concluding remarks in Section 7.

\section{2 | WIND TURBINE}

The wind turbine considered in this paper is the horizontal axis 3-bladed $5 \mathrm{MW}$ reference wind turbine from National Renewable Energy Laboratory (NREL) described in Jonkman et al. ${ }^{26}$ The wind turbine is a variable-speed turbine with a variable-pitch system, including the rotor system and a generator. The simulations in this work are performed with high-fidelity software FAST (Fatigue, Aerodynamics, Structures, and Turbulence) developed by NREL. 


\section{1 | Wind turbine structure}

A wind turbine can be divided into 4 subsystems: the aerodynamics subsystem, mechanical subsystem, electrical subsystem, and actuator subsystem. Wind forces are converted into mechanical torque and thrust on the rotor in the aerodynamic subsystem. The subsystem consists of the drivetrain, tower, and blades. The rotor torque is transferred to the electrical generator in the drivetrain. The tower holds the nacelle and withstands the thrust force, and the blades transform wind forces into torque and thrust. In the generator subsystem, the mechanical energy is converted into electrical energy, and finally, the blade-pitch and generator-torque actuator subsystems are part of the control system.

Figure 1 shows the basic subsystems and their interactions. The control actions are the reference signal to the local actuators that control the pitch angles of the 3 blades $\left(\Theta_{r e f, i}\right)$ and the torque reference $\left(Q_{r e f}\right)$ to the generator controller. The basic degrees

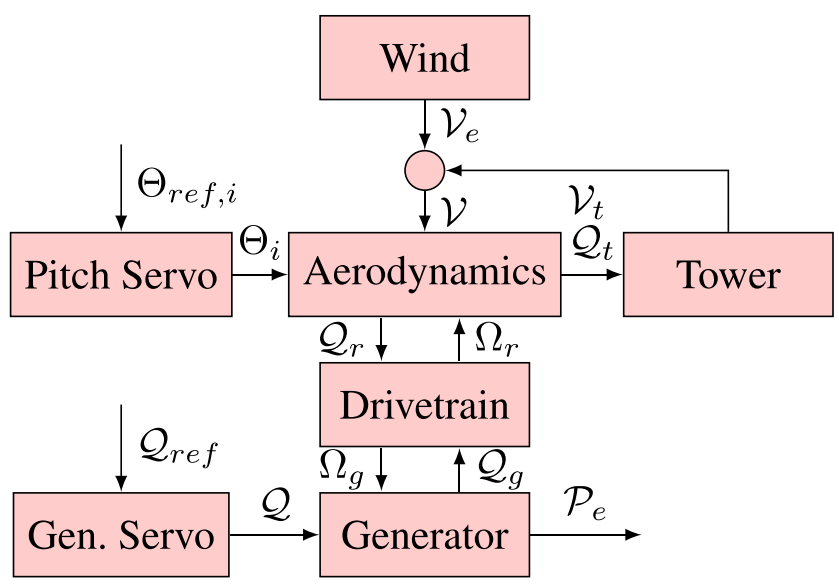

FIGURE 1 Wind turbine subsystems [Colour figure can be viewed at wileyonlinelibrary.com]

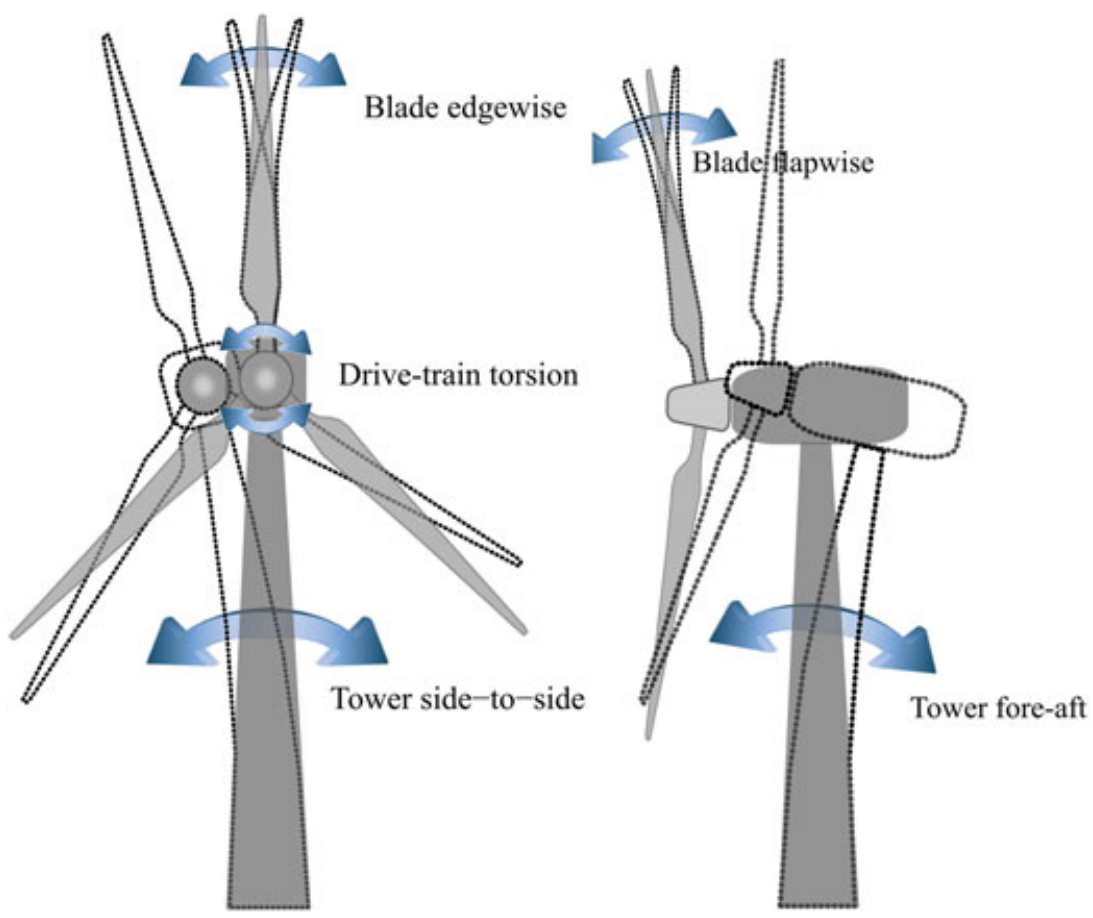

FIGURE 2 Basic degrees of freedom [Colour figure can be viewed at wileyonlinelibrary.com] 


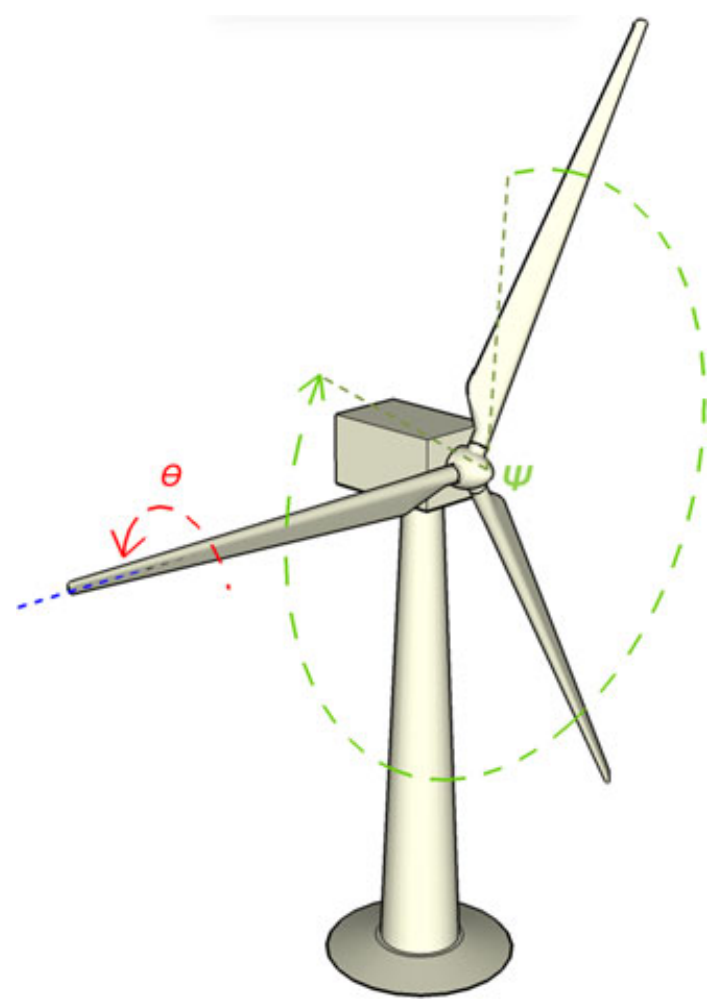

FIGURE 3 The azimuth angle $\Psi$ and pitch angle $\theta$ are shown on a wind turbine rotor [Colour figure can be viewed at wileyonlinelibrary.com]

of freedom that are normally considered in the simulation model are also shown in Figure 2. The azimuth angle $\Psi$ and the pitch angle $\theta$ for a blade are shown in Figure 3.

Modern wind turbines include many sensors for measuring different variables on the turbine. The flap-wise and edge-wise root bending moments for each blade are assumed to be measured together with the azimuth angle. These are the measurements used for fault diagnosis in this work. These measurements are used directly, and no model of the turbine is needed for the fault diagnosis.

\section{2 | Control of wind turbines}

The applied pitch controller for the $5 \mathrm{MW}$ benchmark wind turbine generates the 3 pitch angles $\theta_{i}=\theta_{c}+\Delta \theta_{i}, i=1,2,3$. The controller consists of a collective pitch controller that generates $\theta_{c}$, described in Jonkman et $\mathrm{al}^{26}$ and an individual pitch controller (IPC) that generates $\Delta \theta_{i}, i=1,2,3$. When only a collective pitch controller is applied, $\Delta \theta_{i}=0, i=1,2,3$. In the full-load region, the wind turbine is controlled such that constant power production is obtained. The wind turbine is controlled by using a controller that includes gain-scheduled feedback from the rotor speed to the collective pitch angle to control the generator torque. The IPC system uses flap-wise blade root bending moments via the MBC transform to determine the cyclic behavior of the pitch angles. The cyclic pitch terms are then added to the collective pitch angle.

Wind speed variation affects the speed of the turbine. In many modern types of wind turbines, the speed of the turbine is also controlled by means of, eg, the generator reaction torque. Variation in the turbine speed is not important to fault diagnosis or condition monitoring, but it must be considered.

\section{3 | MULTIBLADE COORDINATE TRANSFORMATION AND SIGNAL MODULATION}

The 2 central elements in the fault diagnosis and condition monitoring method presented in this paper are MBC transformation and signal modulation. These 2 elements are analyzed in detail in the following section. 


\section{1 | Multiblade coordinate transformation}

The wind turbine blade root bending moments are periodic and depend on the azimuth angle. Let us assume, for simplicity of presentation, that the speed of the turbine is constant. We will relax this assumption later. The MBC transformation enables transformation from a rotating frame of reference to a fixed frame of reference. The azimuth angle $\phi_{i}(t)$ of each blade $i=1,2,3$, (for a 3-blade rotor) assuming a constant rotor speed $\Omega$ and equidistant angular spacing between the blades is given by

$$
\phi_{i}(t)=\phi_{0}+\Omega t-(i-1) 2 \pi / 3, \quad i=1,2,3
$$

and renders the MBC transformation as a function of time $t$ rather than the azimuth angle $\phi_{i}(t)$. The angle between the angles applied in the MBC transformation and the rotor blades is given by $\phi_{0}$. The azimuth angles can be combined in a vector given by

$$
\phi(t)=\left[\begin{array}{lll}
\phi_{1}(t) & \phi_{2}(t) & \phi_{3}(t)
\end{array}\right]^{T}
$$

The rotating frame coordinates $\boldsymbol{q}$ and fixed frame coordinates $\boldsymbol{q}^{f}$ have the following relationship:

$$
q^{f}=M q \quad q=M^{-1} q^{f} \quad q=\left[\begin{array}{lll}
q_{1} & q_{2} & q_{3}
\end{array}\right]^{T} \quad q^{f}=\left[\begin{array}{lll}
q_{0}^{f} & q_{c}^{f} & q_{s}^{f}
\end{array}\right]^{T},
$$

where $q_{0}^{f}$ is the collective coordinate, $q_{c}^{f}$ is the cosine-cyclic coordinate, and $q_{s}^{f}$ is the sine-cyclic coordinate (in Park's transformation, these are the DC component, synchronous component, and asynchronous components), see, eg, Stol et al. ${ }^{27}$ The MBC transformation matrices are

$$
\begin{aligned}
M & =\left[\begin{array}{ccc}
\frac{1}{3} & \frac{1}{3} & \frac{1}{3} \\
\frac{2}{3} \cos \left(\phi_{1}(t)\right) & \frac{2}{3} \cos \left(\phi_{2}(t)\right) & \frac{2}{3} \cos \left(\phi_{3}(t)\right) \\
\frac{2}{3} \sin \left(\phi_{1}(t)\right) & \frac{2}{3} \sin \left(\phi_{2}(t)\right) & \frac{2}{3} \sin \left(\phi_{3}(t)\right)
\end{array}\right] \\
M^{-1} & =\left[\begin{array}{lll}
1 & \cos \left(\phi_{1}(t)\right) & \sin \left(\phi_{1}(t)\right) \\
1 & \cos \left(\phi_{2}(t)\right) & \sin \left(\phi_{2}(t)\right) \\
1 & \cos \left(\phi_{3}(t)\right) & \sin \left(\phi_{3}(t)\right)
\end{array}\right] .
\end{aligned}
$$

The actual azimuth angles that are entered into the transformation matrices in Equation 3; thus, the assumption of constant speed, $\Omega$ in Equation 1, can be relaxed. The MBC transformation is shown in Figure 4.

Figure 2 also shows the basic degrees of freedom that are typically considered in the simulation model.

In next section, $q$ will be either the set of measured flap-wise root bending moments or the set of measured edge-wise root bending moments. $q^{f}$ is the equivalent transformed flap-wise or edge-wise root bending moments. Considering the fundamental issues in the MBC transformation and the signal modulation, it is assumed here that the mean value of $q$ is zero in the nominal case.

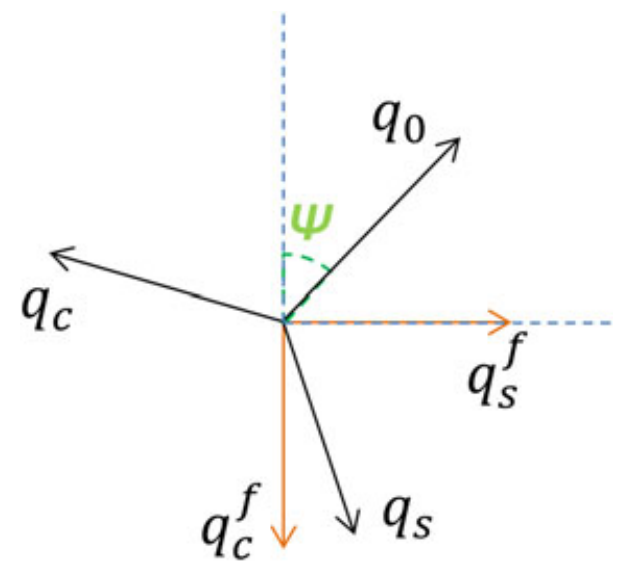

FIGURE 4 The multiblade coordinate transformation, where $\left(q_{0}, q_{c}, q_{s}\right)$ is the rotating frame and $\left(q_{c}^{f}, q_{s}^{f}\right)$ is the fixed frame. In the fixed frame, $q_{o}^{f}$ is 0 [Colour figure can be viewed at wileyonlinelibrary.com] 
The MBC transformation for an isotropic rotor will result in time-invariant quantities; ie, the time-varying azimuth angles $\phi_{i}(t)$ will be transformed into constant angles in the fixed frame. This situation is based on the condition that inputs to the transformation are symmetric. When this condition is not satisfied, the azimuth angles in the fixed frame will not be constant. This issue is analyzed in more detail in the following.

Let the periodic input vector $q$ be given by

$$
q=\left[\begin{array}{l}
q_{1} \\
q_{2} \\
q_{3}
\end{array}\right]=\left[\begin{array}{l}
\sin \left(\phi_{1}(t)-\phi_{0}\right) \\
\sin \left(\phi_{2}(t)-\phi_{0}\right) \\
\sin \left(\phi_{3}(t)-\phi_{0}\right)
\end{array}\right]
$$

where $\phi_{i}(t)$ is given by Equation 1. Further, the MBC transformation matrix $M$ is given by Equation 3. In the fixed frame, $q^{f}$ in Equation 2 is given by

$$
\begin{aligned}
q^{f} & =\left[\begin{array}{c}
q_{0}^{f} \\
q_{c}^{f} \\
q_{s}^{f}
\end{array}\right]=\left[\begin{array}{ccc}
\frac{1}{3} & \frac{1}{3} & \frac{1}{3} \\
\frac{2}{3} \cos \left(\phi_{1}(t)\right) & \frac{2}{3} \cos \left(\phi_{2}(t)\right) & \frac{2}{3} \cos \left(\phi_{3}(t)\right) \\
\frac{2}{3} \sin \left(\phi_{1}(t)\right) & \frac{2}{3} \sin \left(\phi_{2}(t)\right) & \frac{2}{3} \sin \left(\phi_{3}(t)\right)
\end{array}\right]\left[\begin{array}{c}
\sin \left(\phi_{1}(t)-\phi_{0}\right) \\
\sin \left(\phi_{2}(t)-\phi_{0}\right) \\
\sin \left(\phi_{3}(t)-\phi_{0}\right)
\end{array}\right] \\
& =\left[\begin{array}{c}
0 \\
-\sin \left(\phi_{0}\right) \\
\cos \left(\phi_{0}\right)
\end{array}\right] .
\end{aligned}
$$

Equation 5 illustrates that the MBC transformation in the nominal case yields constant states in the fixed frame.

Two types of perturbations are introduced to $q$ : an additive perturbation $\delta_{a}$ and a multiplicative perturbation $\delta_{m}$. First, consider the case of including an additive perturbation to $q$. Let the perturbation $q$ be given by

$$
q=\left[\begin{array}{c}
\sin \left(\phi_{1}(t)-\phi_{0}\right) \\
\sin \left(\phi_{2}(t)-\phi_{0}\right) \\
\sin \left(\phi_{3}(t)-\phi_{0}\right)
\end{array}\right]+\left[\begin{array}{c}
0 \\
0 \\
\delta_{a}
\end{array}\right]
$$

ie, a perturbation at $q_{s}$. In the fixed frame, $q^{f}=M q$ is given by

$$
q^{f}=\left[\begin{array}{c}
0 \\
-\sin \left(\phi_{0}\right) \\
\cos \left(\phi_{0}\right)
\end{array}\right]+\left[\begin{array}{c}
\frac{1}{3} \delta_{a} \\
\frac{2}{3} \delta_{a} \cos \left(\Omega t-\frac{4}{3} \pi+\phi_{0}\right) \\
\frac{2}{3} \delta_{a} \sin \left(\Omega t-\frac{4}{3} \pi+\phi_{0}\right)
\end{array}\right]
$$

Equation 7 illustrates that all components in $q^{f}$ are proportional to the additive perturbation $\delta_{a}$. Furthermore, an additive perturbation yields constant $q_{0}^{f}$ and $1 \mathrm{p}$ variation in $q_{c}^{f}$ and $q_{s}^{f}$.

Now, consider the case in which the input includes a multiplicative perturbation. The perturbation $q$ is then given by

$$
q=\left[\begin{array}{c}
\sin \left(\phi_{1}(t)-\phi_{0}\right) \\
\sin \left(\phi_{2}(t)-\phi_{0}\right) \\
\left(1+\delta_{m}\right) \sin \left(\phi_{3}(t)-\phi_{0}\right)
\end{array}\right]=\left[\begin{array}{c}
\sin \left(\phi_{1}(t)-\phi_{0}\right) \\
\sin \left(\phi_{2}(t)-\phi_{0}\right) \\
\sin \left(\phi_{3}(t)-\phi_{0}\right)
\end{array}\right]+\left[\begin{array}{c}
0 \\
0 \\
\delta_{m} \sin \left(\phi_{3}(t)-\phi_{0}\right)
\end{array}\right]
$$

The perturbation is again placed at $q_{s}$. In the fixed frame, $q^{f}$ is given by

$$
q^{f}=\left[\begin{array}{c}
0 \\
-\sin \left(\phi_{0}\right) \\
\cos \left(\phi_{0}\right)
\end{array}\right]+\left[\begin{array}{c}
\frac{1}{3} \delta_{m} \sin \left(\phi_{3}(t)-\phi_{0}\right) \\
\frac{2}{3} \delta_{m} \cos \left(\phi_{3}(t)\right) \sin \left(\phi_{3}(t)-\phi_{0}\right) \\
\frac{2}{3} \delta_{m} \sin \left(\phi_{3}(t)\right) \sin \left(\phi_{3}(t)-\phi_{0}\right)
\end{array}\right]=\left[\begin{array}{c}
\frac{1}{3} \delta_{m} \sin \left(\Omega t-\frac{4}{3} \pi\right) \\
-\left(1+\frac{1}{3} \delta_{m}\right) \sin \left(\phi_{0}\right)+\frac{1}{3} \delta_{m} \sin \left(2 \Omega t-\frac{2}{3} \pi+\phi_{0}\right) \\
\left(1+\frac{1}{3} \delta_{m}\right) \cos \left(\phi_{0}\right)-\frac{1}{3} \delta_{m} \cos \left(2 \Omega t-\frac{2}{3} \pi+\phi_{0}\right)
\end{array}\right]
$$

Equation 9 illustrates that all components in $q^{f}$ are proportional to the multiplicative perturbation $\delta_{m}$. Further, $\delta_{m}$ yields a $1 \mathrm{p}$ variation of $q_{0}^{f}$ and a $2 p$ variation in $q_{c}^{f}$ and $q_{s}^{f}$. Furthermore, there is a phase shift between the input and the outputs in $q_{c}^{f}$ and $q_{s}^{f}$. The results for various perturbations are shown in Figure 5. 

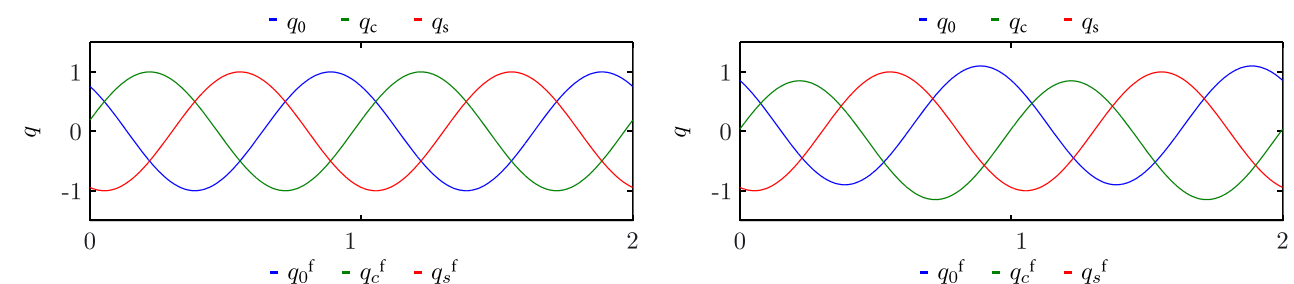

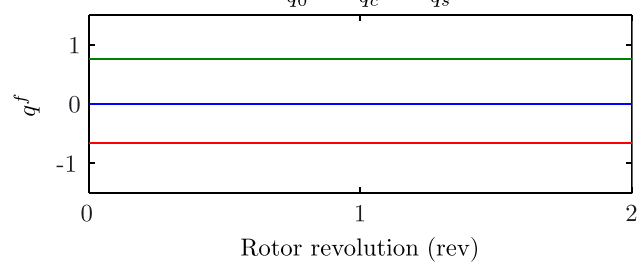

(A) Nominal case

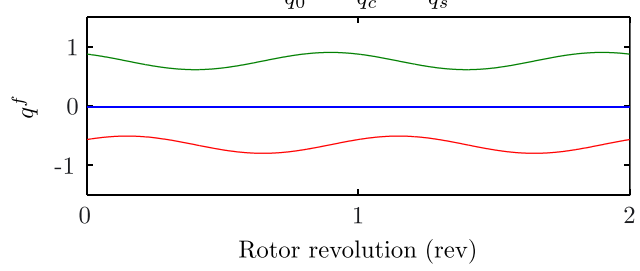

(B) Additive perturbation
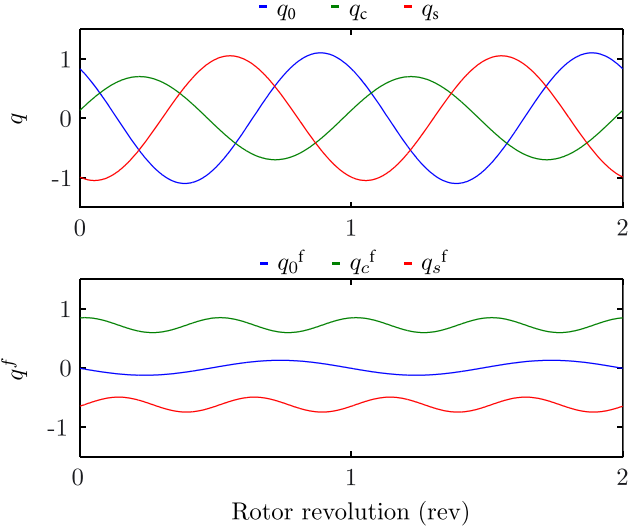

(C) Multiplicative perturbation

FIGURE 5 Multiblade coordinate (MBC) transformation for various perturbations. Note the signatures of different types of faults in the presence of the $1 p$ and $2 p$ components in the MBC transforms [Colour figure can be viewed at wileyonlinelibrary.com]

Faults on the wind turbine rotor will typically result in asymmetries in the rotor. Using the MBC transformation, it is possible to detect asymmetries in the rotor, which is an indirect detection of faults in the system. This is the basis for fault detection and fault isolation in the rotor system, as shown below.

The above 2 types of perturbation are the standard faults or perturbations considered for fault diagnosis. However, for the $\mathrm{MBC}$ transformation, it is also relevant to consider phase perturbation, ie, a phase perturbation in one of the input signals.

In the general, MBC transformation, it is also possible to consider the case in which a phase perturbation is included. This is included for completeness in Appendix A but is not relevant to faults on wind turbines.

\section{2 | Signal modulation}

The above analysis demonstrates that a constant additive perturbation will result in a constant perturbation of $q_{0}^{f}$ and a periodic perturbation with $1 p$ variation in $q_{c}^{f}$ and $q_{s}^{f}$ in the fixed coordinate frame. A constant multiplicative perturbation will yield perturbations with both $1 p$ and $2 p$ variations in the fixed frame.

A modulation of the $q^{f}$ is applied with respect to the $1 p$ and $2 p$ variations to analyze the output of the MBC transformation.

First, consider the additive case in which $q^{f}$ is given by Equation 7, ie, an additive perturbation on $q_{3}$. The constant term in $q_{0}^{f}$ from the additive fault cannot be applied in the following diagnosis, as will be shown later. Thus, this term will not be considered further. The 2 terms in Equation 7 are given by

$$
\left[\begin{array}{c}
q_{c}^{f} \\
q_{s}^{f}
\end{array}\right]=\left[\begin{array}{c}
\frac{2}{3} \delta_{a} \cos \left(\Omega t-\frac{4}{3} \pi+\phi_{0}\right) \\
\frac{2}{3} \delta_{a} \sin \left(\Omega t-\frac{4}{3} \pi+\phi_{0}\right)
\end{array}\right] .
$$


Multiplying $q_{c}^{f}$ and $q_{s}^{f}$ by both $\cos (\Omega t)$ and $\sin (\Omega t)$ gives the following 4 signals:

$$
\left[\left(\begin{array}{c}
q_{c}^{f} \cos (\Omega t) \\
q_{c}^{f} \sin (\Omega t)
\end{array}\right)\right]=\frac{1}{3} \delta_{a}\left[\begin{array}{c}
\left(\begin{array}{c}
\cos \left(\frac{4}{3} \pi-\phi_{0}\right)+\cos \left(2 \Omega t-\frac{4}{3} \pi+\phi_{0}\right) \\
\sin \left(\frac{4}{3} \pi-\phi_{0}\right)+\sin \left(2 \Omega t-\frac{4}{3} \pi+\phi_{0}\right)
\end{array}\right) \\
\left(\begin{array}{c}
q_{s}^{f} \cos (\Omega t) \\
q_{s}^{f} \sin (\Omega t)
\end{array}\right)
\end{array}\right]
$$

The constant terms in Equation 11 are given by

$$
\left.\left[\begin{array}{l}
v_{c, 3,1 p} \\
v_{s, 3,1 p}
\end{array}\right]=\left[\begin{array}{c}
\left(\begin{array}{c}
q_{c}^{f} \cos (\Omega t) \\
q_{c}^{f} \sin (\Omega t)
\end{array}\right) \\
\left(\begin{array}{c}
q_{s}^{f} \cos (\Omega t) \\
q_{s}^{f} \sin (\Omega t)
\end{array}\right)
\end{array}\right]_{\text {constant }}=\frac{1}{3} \delta_{a}\left[\begin{array}{c}
\cos \left(-\frac{4}{3} \pi+\phi_{0}\right) \\
-\sin \left(-\frac{4}{3} \pi+\phi_{0}\right)
\end{array}\right)\right],
$$

where the vector notation is as follows: $v_{i, j, k}$, where $i$ is the signal in the fixed frame, $i=0, c, s ; j$ is the blade number, $j=1,2,3$; and $k$ indicates whether the modulation is with respect to $1 p$ or $2 p\left(v_{0, j, 2 p}\right.$ does not exist, see Appendix B). In the following, $v_{i, j, k}$ will be called the modulation vectors.

Equation 12 yields 2 vectors, $v_{c, 3,1 p}$ and $v_{s, 3,1 p}$, in the complex plane specified by the phase $-\frac{4}{3} \pi+\phi_{0}$. An integration of these signals can be used to detect an additive fault in the system. A simple detection scheme is to use

$$
\varepsilon_{\text {detection }}=\int_{0}^{t}\left(\sqrt{\left(q_{c}^{f} \cos (\Omega t)\right)^{2}+\left(q_{c}^{f} \sin (\Omega t)\right)^{2}}\right) d t \approx \frac{1}{3} \delta_{a} t
$$

that will yield an increasing function for an additive fault (assuming that the fault occurs at $t=0$ ). A CUSUM test method described in, eg, Basseville and Nikiforov ${ }^{4}$ and Gustafsson, ${ }^{5}$ can also be applied. $q_{s}^{f}$ can be applied for detection instead of $q_{c}^{f}$.

Additional information is needed for fault isolation. For the isolation case, the signals are integrated individually. An integration of the single signals in Equation 11 yields the following result:

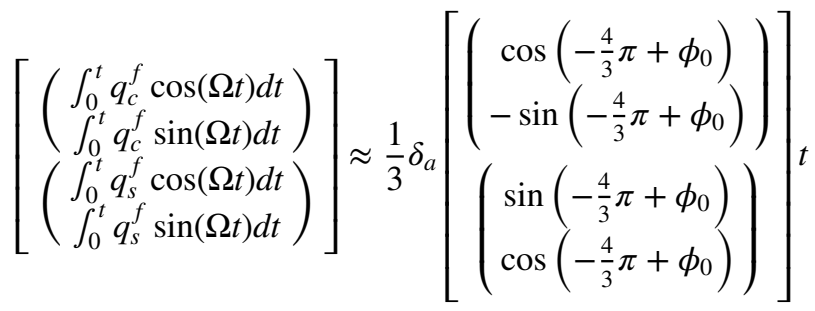

$$
\begin{aligned}
& =\left[\begin{array}{c}
v_{c, 3,1 p} \\
v_{s, 3,1 p}
\end{array}\right] t
\end{aligned}
$$

(assuming that the fault occurred at $t=0$ ). This result yields 2 sets of vectors in the complex plane that depend on the phase $-\frac{4}{3} \pi+\phi_{0}$. Compared with Equation 12, the mean values of the integration yield vectors that are proportional to the 2 sets of vectors in Equation 12. Furthermore, these 2 vectors are orthogonal to each other.

The above modulation and following integration have been performed for a fault in the last input (blade no. 3). Performing these steps on all 3 inputs (blades), we obtain the 3 sets of modulation vectors given in Table B1 in Appendix B. For completeness, $q_{0}^{f}$ has also been included in Table B1. $q_{0}^{f}$ is constant and is not used for fault isolation, as described in the next section.

The input number (blade number) where the additive fault has occurred can be isolated using the directions given in Table B1. There is a phase shift of $\frac{2}{3} \pi$ between the 2 vectors $v_{c, j_{1}, 1 p}$ and $v_{c, j_{2}, 1 p}$ from 2 different blades. The same is the case when using the other modulation vector $v_{s, j, 1 p}$. This yields 2 sets of modulation vectors for isolating an additive fault. 


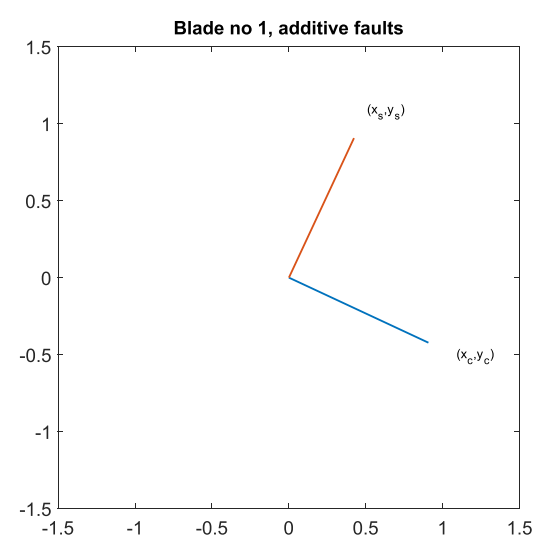

(A) Blade no. 1

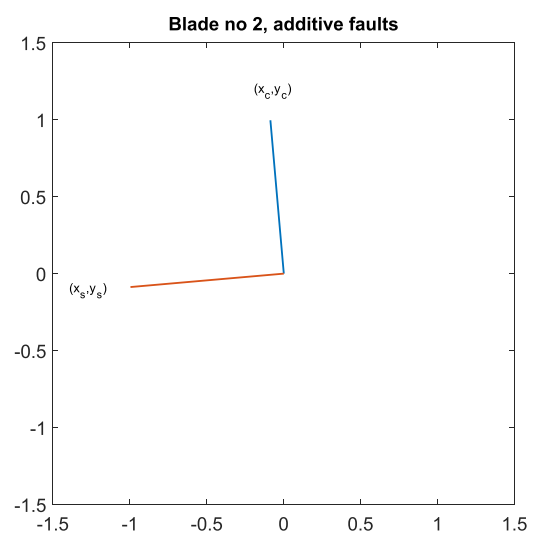

(B) Blade no. 2

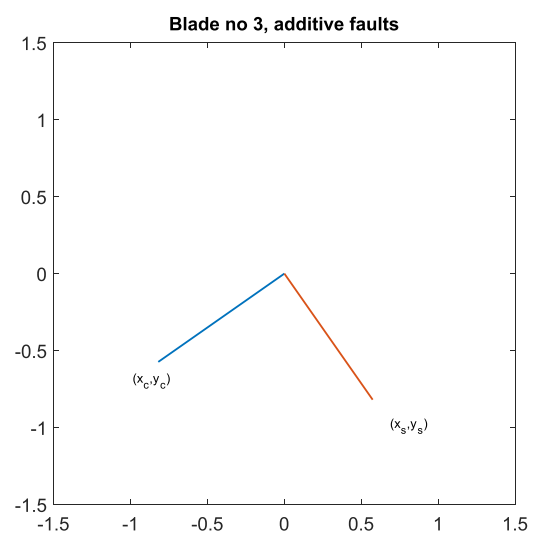

(C) Blade no. 3

FIGURE 6 Modulation vectors after multiblade coordinate transformation for additive faults [Colour figure can be viewed at wileyonlinelibrary.com]

It has previously been shown that a multiplicative fault results in a $1 p$ variation in $q_{0}^{f}$ and $2 p$ variations in $q_{c}^{f}$ and $q_{s}^{f}$. From Equation 9, the 3 terms related to the multiplicative faults in input no. 3 are given by

$$
\left[\begin{array}{c}
q_{0}^{f} \\
q_{c}^{f} \\
q_{s}^{f}
\end{array}\right]=\frac{1}{3} \delta_{m}\left[\begin{array}{c}
\sin \left(\Omega t-\frac{4}{3} \pi\right) \\
\sin \left(2 \Omega t-\frac{2}{3} \pi+\phi_{0}\right) \\
\cos \left(2 \Omega t-\frac{2}{3} \pi+\phi_{0}\right)
\end{array}\right] .
$$

Following the same process as with additive faults, the first term in Equation 15 is then multiplied by $\cos (\Omega t)$ and $\sin (\Omega t)$, and the last 2 terms are multiplied by $\cos (2 \Omega t)$ and $\sin (2 \Omega t)$. This yields the 3 sets of modulation vectors given in Table B2 in Appendix B.

It is again possible to isolate which input (blade) the fault is related to based on the above results.

The 2 sets of modulation vectors shown in Tables B1 and B2 for additive faults and multiplicative faults are shown in Figure 6 and 7 , respectively.

In all results, $\phi_{0}$ is the difference between the angles applied in the MBC transformation and the rotor blades. However, the azimuth angle is measured; thus, $\phi_{0}$ is known.

\section{4 | WIND TURBINE MONITORING AND FAULT DIAGNOSIS}

In the previous section, it was assumed that the mean value of $q$ is 0 in the nominal case, ie, the fault-free case. This assumption is not satisfied for real measurements of the root bending moments. In the real case, there will be an offset in the root bending moments. However, the MBC transformation can also handle the case in which there is a direct offset in the measured moments. 


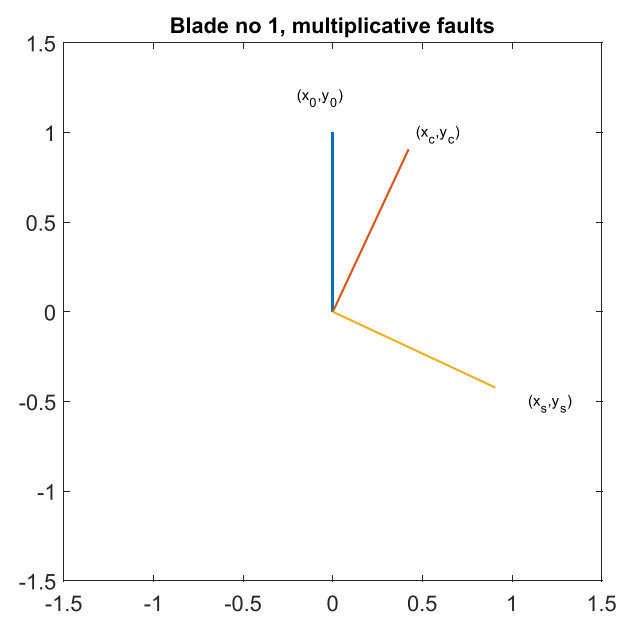

(A) Blade no. 1

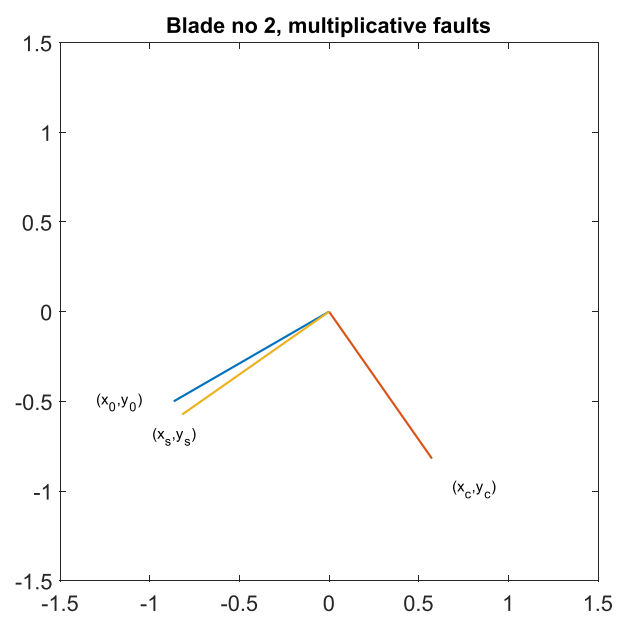

(B) Blade no. 2

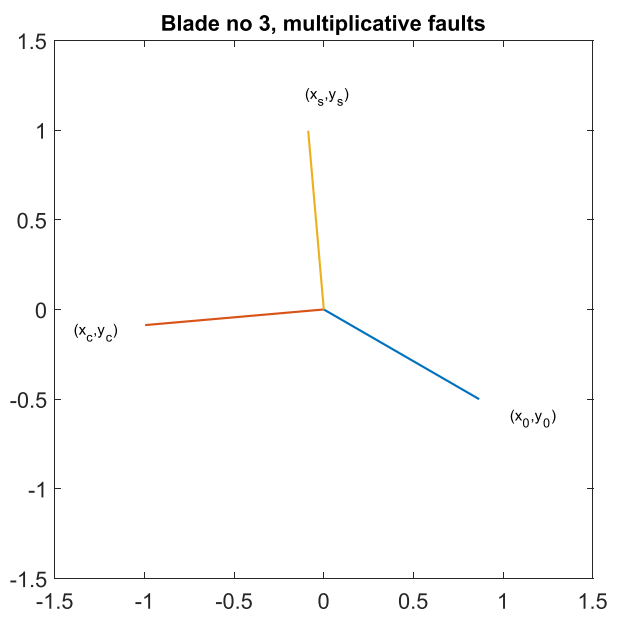

(C) Blade no. 3

FIGURE 7 Modulation vectors after multiblade coordinate transformation for multiplicative faults [Colour figure can be viewed at wileyonlinelibrary.com]

In this case, the $q_{0}^{f}$ component in Equation 5 will be nonzero, as shown below. In this section, an analysis of the offset case is given with respect to additive and multiplicative faults. Furthermore, a method for the compensation of a nonsymmetrical rotor due to production issues will also be described in this section.

\section{1 | Root bending moments with offset}

The central element in the described fault diagnosis method is the MBC transformation considered in the previous section. An analysis of the MBC transformation of root bending moments with an offset is given in the following.

Let a set of real root bending moments be given by

$$
q=q_{\mathrm{off}}\left[\begin{array}{l}
1 \\
1 \\
1
\end{array}\right]+q_{\mathrm{sym}}\left[\begin{array}{c}
\sin \left(\phi_{1}(t)-\phi_{0}\right) \\
\sin \left(\phi_{2}(t)-\phi_{0}\right) \\
\sin \left(\phi_{3}(t)-\phi_{0}\right)
\end{array}\right],
$$

where $q_{\text {off }}$ is the offset of the root bending moments, and $q_{\text {sym }}$ is the symmetrical part of the root bending moments. In the fixed frame, $q^{f}=M q$ is given by

$$
q^{f}=\left[\begin{array}{c}
q_{0}^{f} \\
q_{c}^{f} \\
q_{s}^{f}
\end{array}\right]=\left[\begin{array}{c}
q_{\mathrm{off}} \\
-q_{\mathrm{sym}} \sin \left(\phi_{0}\right) \\
q_{\mathrm{sym}} \cos \left(\phi_{0}\right)
\end{array}\right]
$$


Equation 17 illustrates that the offset part yields a nonzero $q_{0}^{f}$. Further, the 2 other elements in Equation 17 are scaled with the value of the symmetrical part $q_{\text {sym }}$ of the root bending moments. This is in line with the MBC transformation.

In the case of an additive fault, the root bending moments in the rotating coordinate system are given by

$$
q=q_{\text {off }}\left[\begin{array}{l}
1 \\
1 \\
1
\end{array}\right]+q_{\mathrm{sym}}\left[\begin{array}{c}
\sin \left(\phi_{1}(t)-\phi_{0}\right) \\
\sin \left(\phi_{2}(t)-\phi_{0}\right) \\
\sin \left(\phi_{3}(t)-\phi_{0}\right)
\end{array}\right]+\left[\begin{array}{c}
0 \\
0 \\
\delta_{a}
\end{array}\right]
$$

for an additive fault at blade no. 3. In the fixed frame, $q^{f}$ is given by

$$
\begin{aligned}
q^{f} & =\left[\begin{array}{c}
q_{\mathrm{off}} \\
-q_{\mathrm{sym}} \sin \left(\phi_{0}\right) \\
q_{\mathrm{sym}} \cos \left(\phi_{0}\right)
\end{array}\right]+\left[\begin{array}{c}
\frac{1}{3} \delta_{a} \\
\frac{2}{3} \delta_{a} \cos \left(\Omega t-\frac{4}{3} \pi+\phi_{0}\right) \\
\frac{2}{3} \delta_{a} \sin \left(\Omega t-\frac{4}{3} \pi+\phi_{0}\right)
\end{array}\right] \\
& =q_{\text {constant }}^{f}+q_{\text {additive }}^{f} .
\end{aligned}
$$

As noted in the previous section, $q_{0}^{f}$ should not be used for detection and isolation of additive faults because $q_{\text {off }}$ (and $q_{\text {sym }}$ ) is unknown. A change in $q_{0}^{f}$ can be detected by using a statistical test. However, a wind turbine will not be operating at a steady-state point, but the operation point will depend directly on the wind speed. Thus, the resulting $q_{\text {off }}$ and $q_{\text {sym }}$ will not be constant but also depend on the wind speed. The consequence is that $q_{0}^{f}$ in Equation 19 will not have a constant mean value, and therefore, detection of a change in the mean value due to an additive fault will be extremely difficult. Equation 19 demonstrates that the two $1 p$ components are independent of $q_{\mathrm{off}}$ and $q_{\mathrm{sym}}$ and will not be affected in the fault diagnosis.

The case of multiplicative faults is slightly more complex than that of additive faults. Let $q$ with a multiplicative fault at blade no. 3 and an offset be given by

$$
\begin{aligned}
q & =q_{\text {off }}\left[\begin{array}{c}
1 \\
1 \\
1+\delta_{m}
\end{array}\right]+q_{\mathrm{sym}}\left[\begin{array}{c}
\sin \left(\phi_{1}(t)-\phi_{0}\right) \\
\sin \left(\phi_{2}(t)-\phi_{0}\right) \\
\left(1+\delta_{m}\right) \sin \left(\phi_{3}(t)-\phi_{0}\right)
\end{array}\right] \\
& =q_{\text {off }}\left[\begin{array}{l}
1 \\
1 \\
1
\end{array}\right]+\left[\begin{array}{c}
0 \\
0 \\
q_{\text {off }} \delta_{m}
\end{array}\right]+q_{\text {sym }}\left[\begin{array}{c}
\sin \left(\phi_{1}(t)-\phi_{0}\right) \\
\sin \left(\phi_{2}(t)-\phi_{0}\right) \\
\left(1+\delta_{m}\right) \sin \left(\phi_{3}(t)-\phi_{0}\right)
\end{array}\right] .
\end{aligned}
$$

Equation 20 demonstrates that a multiplicative fault also results in an additive fault depending on the offset in the root bending moments. The term in Equation 20 from the additive fault related to the offset depends on the unknown gain $q_{\text {off }}$, and the term from the multiplicative fault depends on the other unknown gain $q_{\mathrm{sym}}$. After the MBC transformation, $q^{f}$ is given by

$$
\begin{aligned}
q^{f}= & {\left[\begin{array}{c}
q_{\text {off }} \\
-q_{\text {sym }}\left(1+\frac{1}{3} \delta_{m}\right) \sin \left(\phi_{0}\right) \\
q_{\text {sym }}\left(1+\frac{1}{3} \delta_{m}\right) \cos \left(\phi_{0}\right)
\end{array}\right]+q_{\text {off }}\left[\begin{array}{c}
\frac{1}{3} \delta_{m} \\
\frac{2}{3} \delta_{m} \cos \left(\Omega t-\frac{4}{3} \pi+\phi_{0}\right) \\
\frac{2}{3} \delta_{m} \sin \left(\Omega t-\frac{4}{3} \pi+\phi_{0}\right)
\end{array}\right] } \\
& +q_{\text {sym }}\left[\begin{array}{c}
\frac{1}{3} \delta_{m} \sin \left(\Omega t-\frac{4}{3} \pi\right) \\
\frac{1}{3} \delta_{m} \sin \left(2 \Omega t-\frac{2}{3} \pi+\phi_{0}\right) \\
-\frac{1}{3} \delta_{m} \cos \left(2 \Omega t-\frac{2}{3} \pi+\phi_{0}\right)
\end{array}\right] \\
= & q_{\text {constant }}^{f}+q_{\text {additive }}^{f}+q_{\text {multiplicative }}^{f} .
\end{aligned}
$$

When an offset is included in the root bending moments, the multiplicative fault case becomes more complex than in the simple case analyzed in the previous section. The offset will still result in a $1 p$ component in $q_{0}^{f}$ and $2 p$ components for $q_{c}^{f}$ and $q_{s}^{f}$ given by $q_{\text {multiplicative }}^{f}$ in Equation 21. Furthermore, an offset in the root bending moments, together with a multiplicative fault, will also result in two $1 p$ elements in $q_{c}^{f}$ and $q_{s}^{f}$. Altogether, the only change in $q^{f}$ is that $q_{c}^{f}$ and $q_{s}^{f}$ will also include $1 p$ elements that can be applied for detection and isolation. The simulations presented in Section 5 demonstrate that multiplicative faults will result in $1 p$ effects in $q_{c}^{f}$ and $q_{s}^{f}$. Furthermore, these $1 p$ effects in $q_{c}^{f}$ and $q_{s}^{f}$ can be reasonably large compared with the $2 p$ effects in $q_{c}^{f}$ and $q_{s}^{f}$ because of the ratio between $q_{\text {off }}$ and $q_{\text {sym }}$. 
For additive faults, it is difficult to use $q_{0}^{f}$ for fault detection because it depends on the unknown $q_{\text {off }}$. This is not a problem in the multiplicative fault case, where $1 p$ and $2 p$ elements depend on the unknown $q_{\text {off }}$ and $q_{\text {sym }}$. The $1 p$ and $2 p$ elements will only occur when there is a multiplicative fault. The detection of $1 p$ and $2 p$ elements is independent of knowledge about the values of $q_{\text {off }}$ and $q_{\text {sym }}$.

\section{2 | Compensation for a nonsymmetrical rotor system}

A complete new rotor system for a wind turbine will not be precisely symmetrical. It is not possible to produce a rotor without any variations affecting the fault diagnosis. The result of such an asymmetrical rotor system is that some or all of the modulation vectors will be nonzero. Let the modulation vectors related to the asymmetry in the rotor system from production be given by

$$
v_{i, j, k, n o m}, \quad i=[0, c, s], \quad j=[1,2,3], \quad k=[1 p, 2 p] .
$$

It is then possible to compensate for the asymmetry in the integration of the modulation vectors as shown in Equation 14. The integration in Equation 14 can be modified in the following simple manner for an additive fault on blade $j$ :

$$
\left[\begin{array}{l}
v_{c, j, 1 p} \\
v_{s, j, 1 p}
\end{array}\right] t \approx\left[\begin{array}{c}
\left(\begin{array}{c}
\int_{0}^{t} q_{c}^{f} \cos (\Omega t) d t \\
\int_{0}^{t} q_{c}^{f} \sin (\Omega t) d t
\end{array}\right) \\
\left(\begin{array}{c}
\int_{0}^{t} q_{s}^{f} \cos (\Omega t) d t \\
\int_{0}^{t} q_{s}^{f} \sin (\Omega t) d t
\end{array}\right)
\end{array}\right]-\left[\begin{array}{l}
v_{c, j, 1 p, \text { nom }} \\
v_{s, j, 1 p, \text { nom }}
\end{array}\right] t .
$$

It is possible to apply a statistical test method instead of using integration, as in Equation 22.

From the above, it seems to be reasonably straightforward to compensate for asymmetries related to the production of the rotor system. However, there is an additional element related to $v_{i, j, k, n o m}$ that must be considered in the compensation. This additional element is needed because the nominal modulation vectors given by $v_{i, j, k, n o m}$ are not constant but will depend on $q_{\text {off }}$ and $q_{\mathrm{sym}}$. As noted above, $q_{\mathrm{off}}$ and $q_{\mathrm{sym}}$ are not constant but depend on the operation point. These 2 parameters can be estimated and compensate for in the modulation vectors. Estimation of $q_{\text {off }}$ and $q_{\text {sym }}$ will not be considered in this paper.

\section{3 | Actuator and blade faults}

The analysis above is related to sensor faults. A sensor fault can be both detected and isolated. However, faults other than sensor faults can occur in the rotor system, such as actuator faults and blade faults. These faults can also be detected and isolated. Detecting the faults is possible as long as the faults result in an asymmetry in the rotor system. Then, $1 p$ and or $2 p$ effects can be detected in the MBC-transformed signals depending on the faults.

It is also possible to isolate both actuator faults and blade faults. An actuator fault will yield an offset in the pitch for one of the blades. A change in the pitch for a blade will result in a change in both the flap-wise and edge-wise moments. Thus, the effect will be measured in both the flap-wise and edge-wise sensors. After the MBC transformations, the effect from an actuator fault can be observed in both sets of transformed moments. The signature of an actuator fault will be different from the signatures of sensor faults, which enables actuator faults to be isolated from sensor faults. Furthermore, in the same manner as for sensor faults, it is also possible to isolate which actuator is faulty and to determine the type of fault.

The last type of fault is a blade fault. Here, only faults caused by a change in the blade mass are considered. A blade fault caused by a change in the mass is a multiplicative fault. Such a blade fault will also result in signatures in the 2 sets of MBC-transformed moments. As will be shown in Section 5 on the simulations, these signatures from blade faults are also unique; thus, it is possible to isolate actuator faults and sensor faults. Finally, other blade faults may also be relevant. In the case of other types of blade faults, there is no guarantee that it will be possible to isolate these faults from other blade faults or actuator faults.

When a pitch controller is applied to the turbine, it is expected to minimize the effect of faults in the controlled outputs. A pitch controller will not simplify the fault diagnosis. Moreover, the fault diagnosis method described in this paper does not use the control signals. Thus, the information from the control signals caused by the faults is not available for fault diagnosis. In wind turbines, the power production and rotor speed are controlled. The controller will, if possible, keep the power constant or optimize it despite faults in the rotor system. For wind speeds above the rated speed, a pitch fault on 1 blade will be compensated by a minor change in the pitch angles of all blades such that the power production is kept constant. The following fault diagnosis will then only be minimally affected by the feedback controller. 


\section{5 | SIMULATION EXAMPLE}

In this section, the described method will be applied to a simulation of a wind turbine exposed to 2 different faults: actuator faults and blade faults. The method for fault diagnosis will be illustrated in connection to high-fidelity simulation software, which will be described below.

This section is organized as follows. In the first section, we introduce the simulation software and data for the applied wind turbine. The considered pitch and blade faults are briefly described in Section 5.2. Simulation results are shown in Section 5.3, including the fault-free case and 2 faulty cases.

\section{1 | Simulation software}

Simulations are performed to test the described algorithm. The simulations of the behavior of wind turbine models are as close to the behavior of real wind turbines as possible. The simulation model can include several degrees of freedom and nonlinearity that can be modelled mathematically, eg, blade flexibility, tower movement, tower shadow, stochastic wind profile, and turbulence. In this paper, the FAST ${ }^{28}$ simulation software developed at the NREL, is used. FAST is a comprehensive, high-fidelity aero-elastic simulator that is capable of simulating 2-and 3-bladed horizontal-axis wind turbines. The $5 \mathrm{MW}$ reference wind turbine is used as the plant. The main data for the wind turbine are given in Table 1. A more detailed description of the reference wind turbine is provided in Jonkman et al. ${ }^{26}$

The $5 \mathrm{MW}$ wind turbine is controlled by the baseline PI gain scheduled from Jonkman et al. ${ }^{26}$

\section{2 | Fault description}

In the following, we will consider 2 types of faults on wind turbines: mass imbalance faults and additive faults on the blade pitch actuator.

For the mass imbalance fault case, a $2 \%$ percent increase in the mass of the faulty blade has been simulated. Simulations with a $1 \%$ increase in the mass of the blade were also tested. The only difference is a longer detection time for the latter case. Such a fault can be caused by a fracture in a blade, through which water penetrates the blade, changing its mass.

The other fault considered here is an additive actuator fault. The additive fault values are \pm 1 degree in the simulations. Such faults can represent, eg, a constant bias or offset on an actuator or incorrect mounting of a blade (in connection with the production). A further description of possible faults can be found in the benchmark wind turbine model in Odgaard et al. ${ }^{21}$

In summary, the values for the different faults are given in Table 2.

TABLE 1 Data for the 5 MW National Renewable Energy Laboratory wind turbine

\begin{tabular}{ll} 
Rating & \multicolumn{1}{c}{$\mathbf{5} \mathbf{M W}$} \\
\hline Rotor orientation, configuration & Upwind, 3 blades \\
Control & Variable speed \\
Drive-train & High speed, multiple-stage gearbox \\
Rotor, hub diameter & $126 \mathrm{~m}, 3 \mathrm{~m}$ \\
Hub hight & $90 \mathrm{~m}$ \\
Cut-in, rated, cut-out wind speed & $3 \mathrm{~m} / \mathrm{s}, 11.4 \mathrm{~m} / \mathrm{s}, 25 \mathrm{~m} / \mathrm{s}$ \\
Cut-in, rated rotor speed & $6.9 \mathrm{rpm}, 12.1 \mathrm{rpm}$ \\
Rated tip speed & $80 \mathrm{~m} / \mathrm{s}$ \\
Rotor mass & $110.000 \mathrm{~kg}$ \\
Nacelle mass & $240.000 \mathrm{~kg}$ \\
Tower mass & $347.460 \mathrm{~kg}$ \\
\hline
\end{tabular}

TABLE 2 Fault description

\begin{tabular}{ll} 
Fault Types & Fault Value \\
\hline Additive fault on blade pitch actuator & \pm 1 degree \\
Mass imbalance & $1 \%$ and $2 \%$ \\
\hline
\end{tabular}




\section{3 | Simulation results}

Different simulations were conducted using the standard $5 \mathrm{MW}$ wind turbine in FAST. The simulations were conducted in the full-load region. The simulations shown focus on the 2 faults described above. First, the fault-free case is considered.

\subsection{1 | Fault-free case}

The simulation results are shown in the following figures. Figures 8 to 11 show a simulation scenario with a stochastic wind speed, including turbulence with a mean value above the rated wind speed. A collective pitch controller has been applied in these

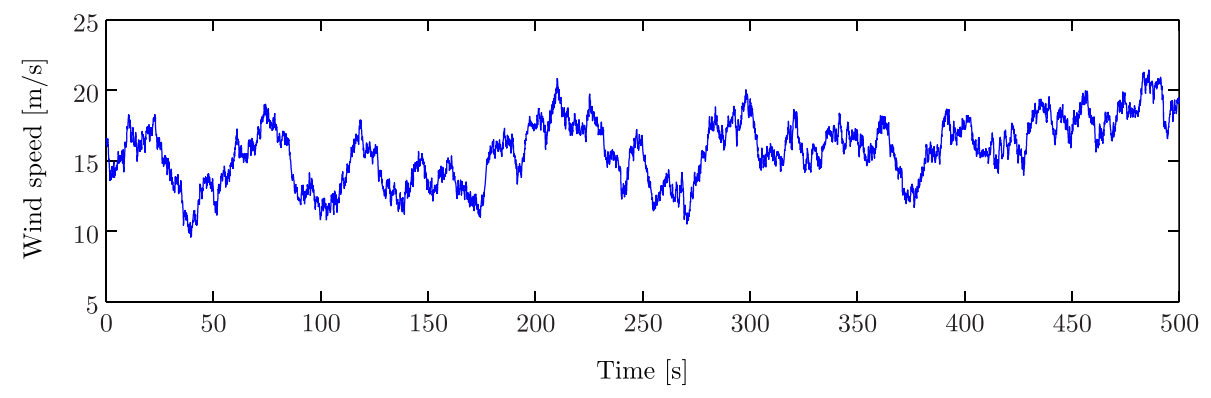

FIGURE 8 Wind speed [Colour figure can be viewed at wileyonlinelibrary.com]

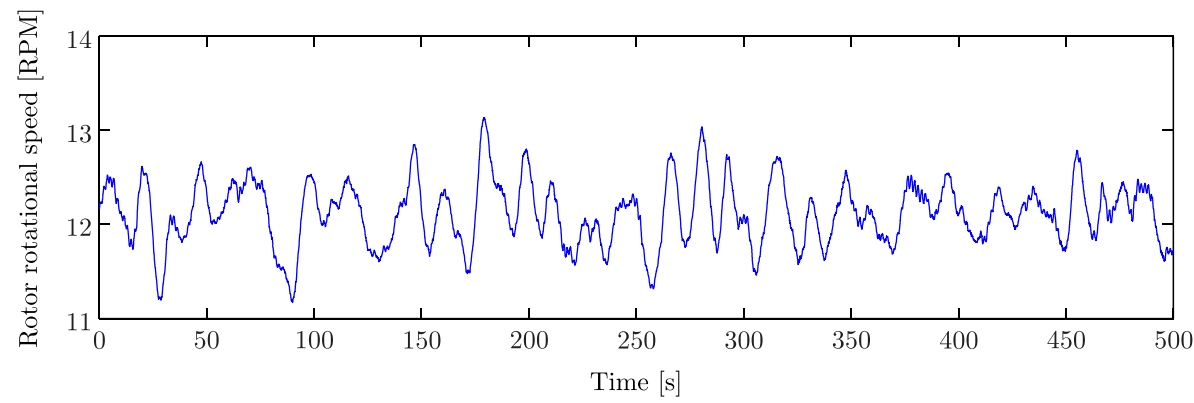

FIGURE 9 Rotational speed of the rotor, fault-free scenario [Colour figure can be viewed at wileyonlinelibrary.com]

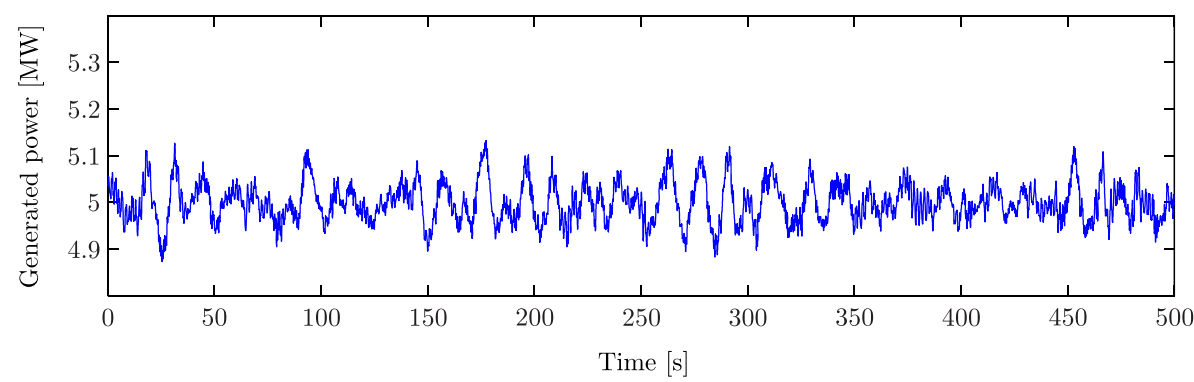

FIGURE 10 Generated power, fault-free scenario [Colour figure can be viewed at wileyonlinelibrary.com]

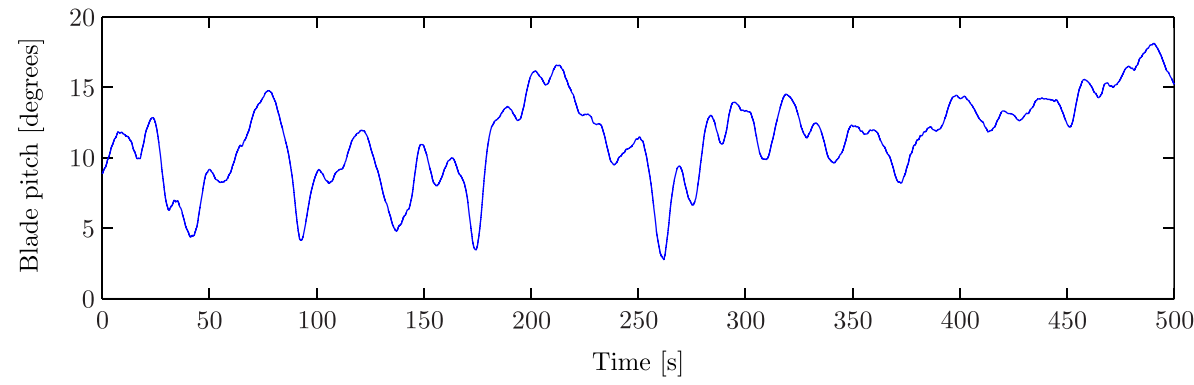

FIGURE 11 Blade pitch, fault-free scenario [Colour figure can be viewed at wileyonlinelibrary.com] 
simulations. Figure 8 shows the applied stochastic wind speed. Figure 8 shows the rotational speed of the rotor, and the generated power is shown in Figure 10. In Figure 11, the blade pitch is shown. All of these simulations are shown for the fault-free case.

The edge-wise and flap-wise root bending moments for 1 blade in the fault-free case are shown in Figures 12 and 13.

The 3 MBC (Coleman) transformed signals for the fault-free case for the flap-wise root bending moment sensors are shown in Figures 14 to 16.

As it can be seen from Figures 14 to 16, the MBC-transformed moments are not constant, as expected in the fault-free case. Thus, a fault cannot be detected directly on the basis of the MBC-transformed moments. Additionally, if the modulation technique described in Section 3.2 is applied, then faults in the rotor system can be detected and isolated. Using the 2 sets of root bending moment sensors, considering both $1 p$ and $2 p$ signals in the MBC-transformed signals, results in 12 sets of vectors in the complex plane that can be used for detection and isolation.

However, it is not possible to show these 12 sets of modulation vectors directly, as noted previously, as each real modulation vector consists of a constant part and periodic part. Instead, the integration over time of the modulation vectors can be observed.

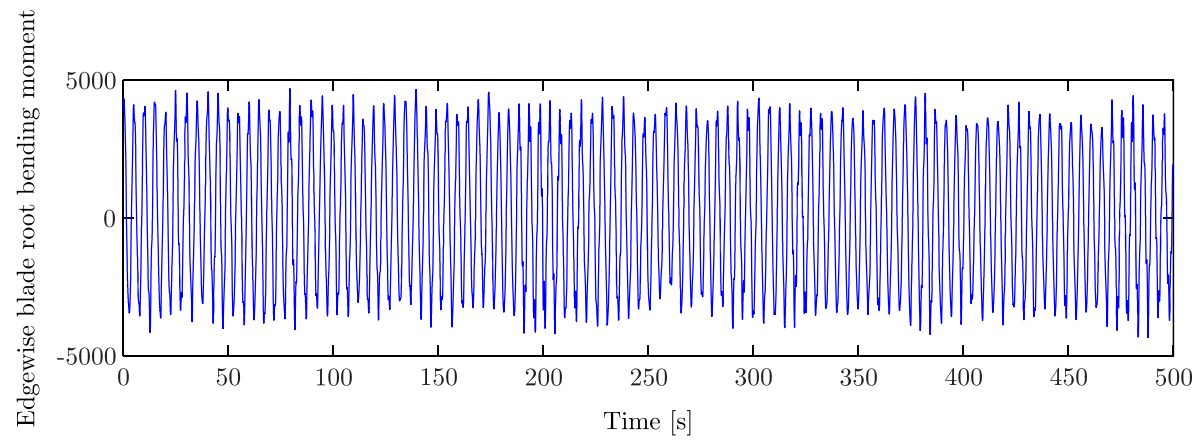

FIGURE 12 Edge-wise root bending moment (measured in $\mathrm{kNm}$ ) for 1 blade, fault-free scenario [Colour figure can be viewed at wileyonlinelibrary.com]

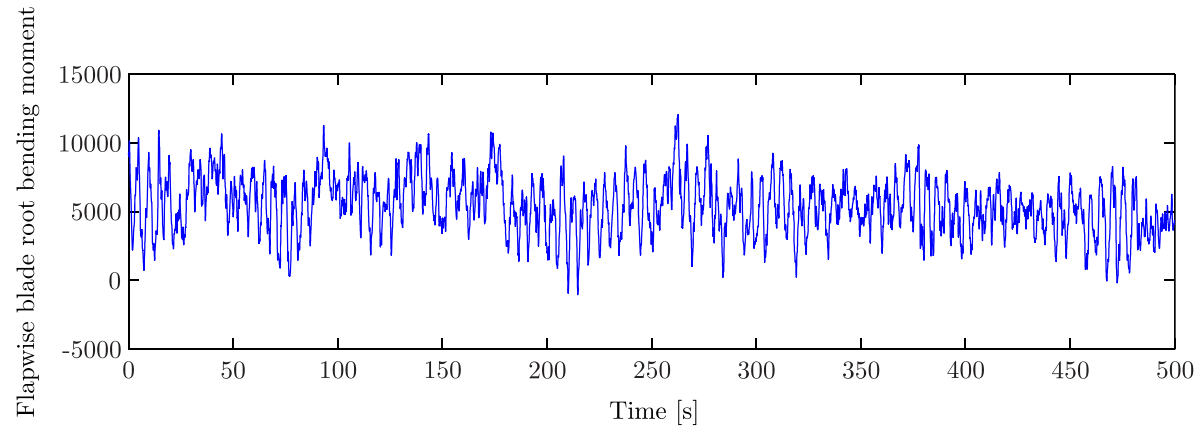

FIGURE 13 Flap-wise root bending moment (measured in $\mathrm{kNm}$ ) for 1 blade, fault-free scenario [Colour figure can be viewed at wileyonlinelibrary.com]

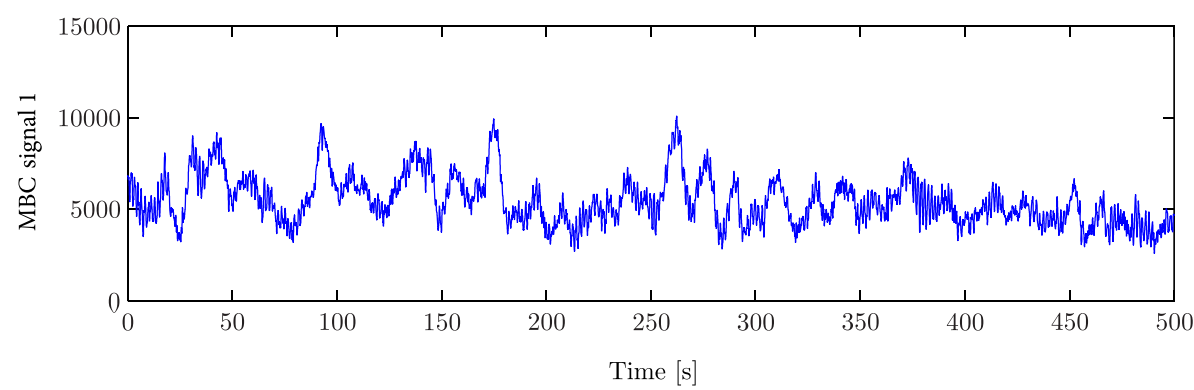

FIGURE 14 The $q_{0}^{f}$ signal after the multiblade coordinate (MBC) transformation, fault-free scenario [Colour figure can be viewed at wileyonlinelibrary.com] 


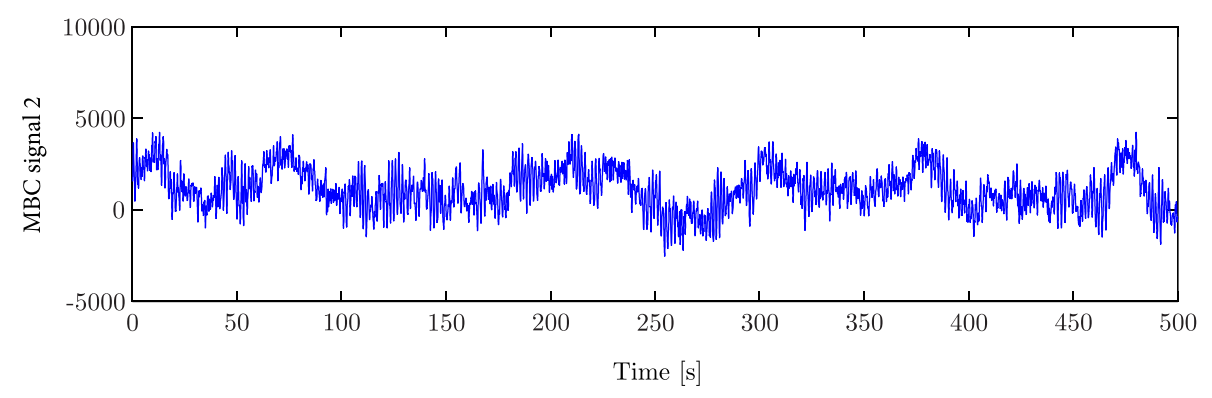

FIGURE 15 The $q_{c}^{f}$ signal after the multiblade coordinate (MBC) transformation, fault-free scenario [Colour figure can be viewed at wileyonlinelibrary.com]

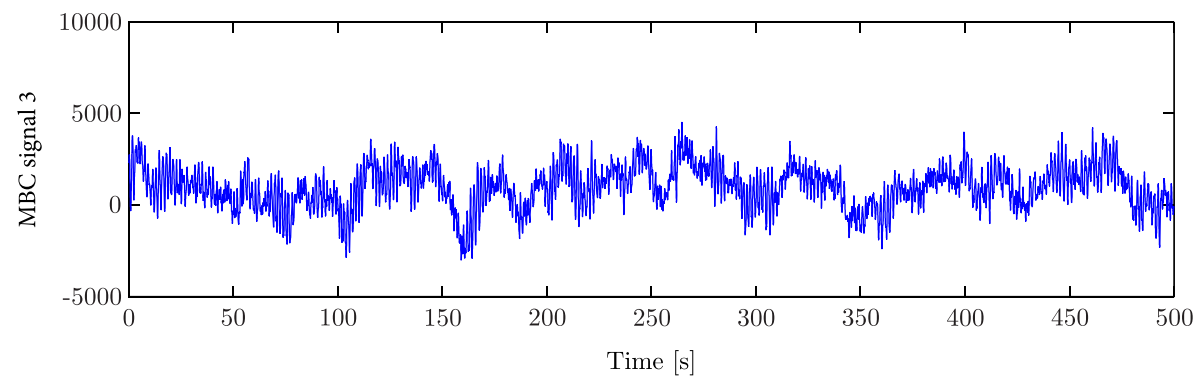

FIGURE 16 The $q_{s}^{f}$ signal after the multiblade coordinate (MBC) transformation, fault-free scenario [Colour figure can be viewed at wileyonlinelibrary.com]

These integrated modulation vectors are given by

$$
\left[\begin{array}{l}
V_{0,1 p} \\
V_{c, 1 p} \\
V_{s, 1 p}
\end{array}\right]=\left[\begin{array}{c}
\left(\begin{array}{c}
\int_{0}^{t} q_{0}^{f} \cos (\Omega t) d t \\
\int_{0}^{t} q_{0}^{f} \sin (\Omega t) d t
\end{array}\right) \\
\left(\begin{array}{c}
\int_{0}^{t} q_{c}^{f} \cos (\Omega t) d t \\
\int_{0}^{t} q_{c}^{f} \sin (\Omega t) d t
\end{array}\right) \\
\left(\begin{array}{c}
\int_{0}^{t} q_{s}^{f} \cos (\Omega t) d t \\
\int_{0}^{t} q_{s}^{f} \sin (\Omega t) d t
\end{array}\right)
\end{array}\right]
$$

for the $1 p$ modulation and

for the $2 p$ modulation.

$$
\left[\begin{array}{l}
V_{0,2 p} \\
V_{c, 2 p} \\
V_{s, 2 p}
\end{array}\right]=\left[\begin{array}{c}
\left(\begin{array}{c}
\int_{0}^{t} q_{0}^{f} \cos (2 \Omega t) d t \\
\int_{0}^{t} q_{0}^{f} \sin (2 \Omega t) d t
\end{array}\right) \\
\left(\begin{array}{c}
\int_{0}^{t} q_{c}^{f} \cos (2 \Omega t) d t \\
\int_{0}^{t} q_{c}^{f} \sin (2 \Omega t) d t
\end{array}\right) \\
\left(\begin{array}{c}
\int_{0}^{t} q_{s}^{f} \cos (2 \Omega t) d t \\
\int_{0}^{t} q_{s}^{f} \sin (2 \Omega t) d t
\end{array}\right)
\end{array}\right]
$$

\subsection{2 | Mass imbalance}

Next, we present simulations with mass imbalances. Here, all 12 (see Equations 24 and (25)) sets of signals are shown. The simulation is in the full-load region, and an individual pitch control from Bossanyi ${ }^{29}$ is applied. This could reduce the signature of a blade fault. Simulations with mass imbalance on 1 blade are shown in Niemann et al, ${ }^{30}$ but here, a collective pitch controller is applied. In the following figures, Figures 17 to 20, a mass imbalance on 1 blade is simulated and compared with the fault-free case (the blue curves). The figures have been scaled such that they all have the same axis, which makes them easier to compare.

Figure 17 shows the integrated $1 p$ modulation signals using the edge-wise moments. Only minor variations can be observed in the case of a mass imbalance on 1 blade. In Figure 18, the equivalent integrated modulation signals are shown for the flap-wise moments. A clear change in the integrated modulation signal $V_{0,1 p}$ (mean value) can be observed in the case of a mass imbalance in 1 blade. This follows the theory that there will be a $1 p$ effect in the mean signal for multiplicative faults. 


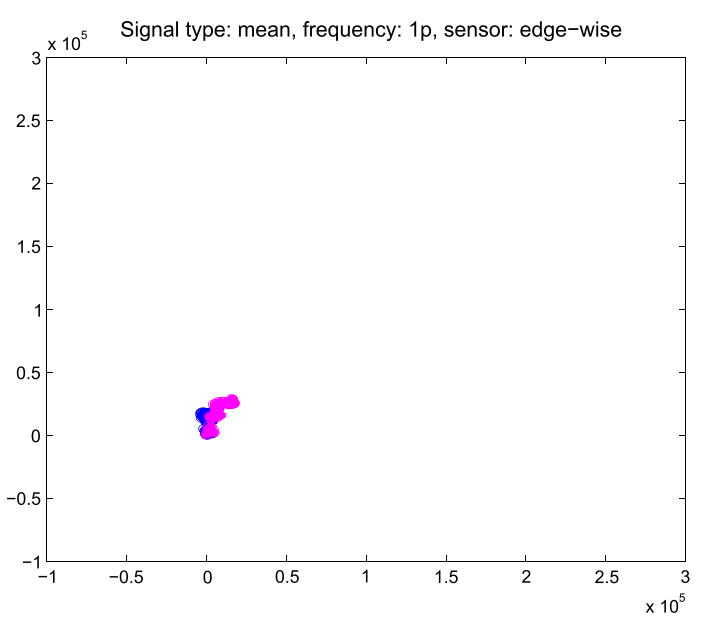

(A) $V_{0,1 p}$

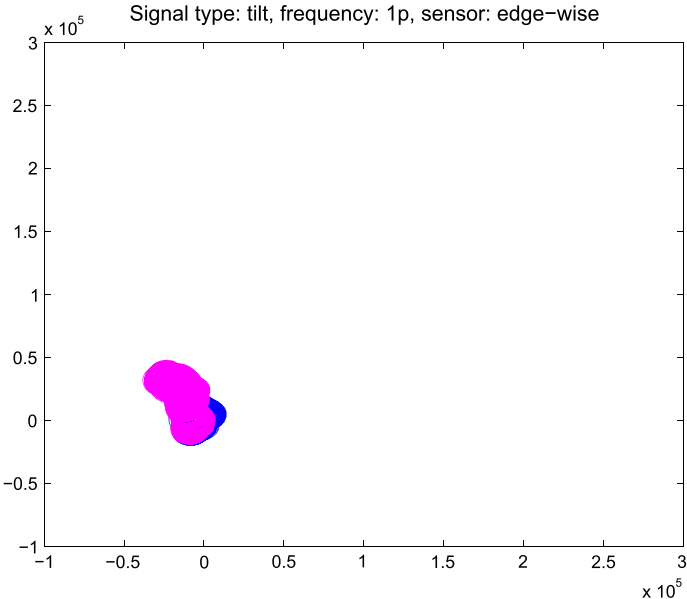

(B) $V_{c, 1 p}$

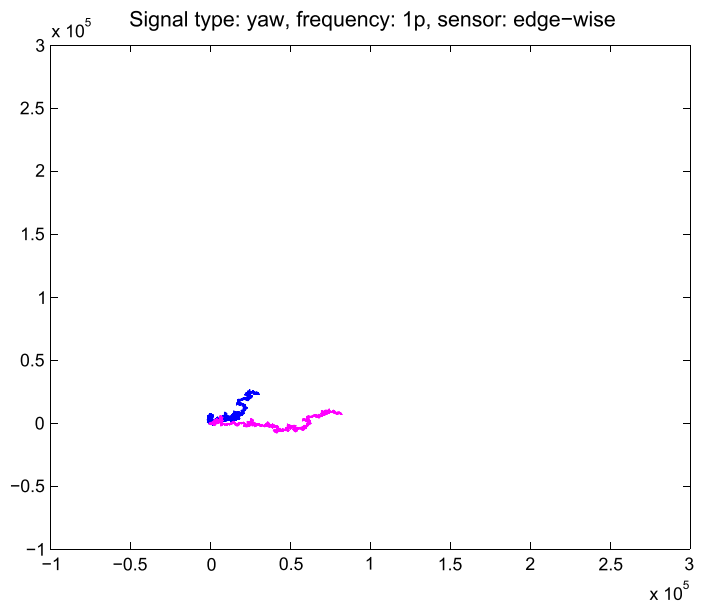

(C) $V_{s, 1 p}$

FIGURE 17 The integrated $1 p$ modulation signals for the edge-wise moments for mass imbalance on 1 blade. The blue curve is for the fault-free case [Colour figure can be viewed at wileyonlinelibrary.com]

In the last 2 figures, Figures 19 and 20, the effects from the integrated $1 p$ modulations used are shown for the 2 sets of edge-wise and flap-wise moment sensors. These 2 sets of plots illustrate that the tilt and yaw $\left(V_{c, 2 p}\right.$ and $\left.V_{s, 2 p}\right)$ vectors deviate away from the nominal curves when the edge-wise sensors are used, indicating that a fault occurred. It is not possible to see any effect of mass imbalance in a blade for the signals related to the flap-wise sensors.

These results agree with the theory that it may be possible to see a $1 p$ effect in the mean values and $2 p$ effects in the other signals, as is shown in these 4 figures. Furthermore, a mass imbalance in 1 blade gives a signature in 3 of the 12 different modulation signals. This will cause a direct isolation of mass imbalance faults from other types of faults in the system. The isolability of the different faults will be discussed further below.

\subsection{3 | Additive actuator fault}

The other type of faults that will be considered here is additive actuator faults. The simulations are still conducted in the full-load region. In this case, a collective pitch controller is applied. Furthermore, instead of showing the result for only 1 blade, the result of faults on all 3 blades are shown. However, only 1 actuator fault is included in each simulation. The simulation results are shown in Figures 21 and 22 for the integrated $1 p$ modulation signals. The integrated $2 p$ modulation signals are not shown because they are reasonably small.

The results shown in Figures 21 and 22 are in line with the theory that there is no effect of the additive actuator faults in the mean signals ( $V_{0,1 p}$ is close to 0$)$ but that there are instead some effects in the other signals. The effect of additive actuator 


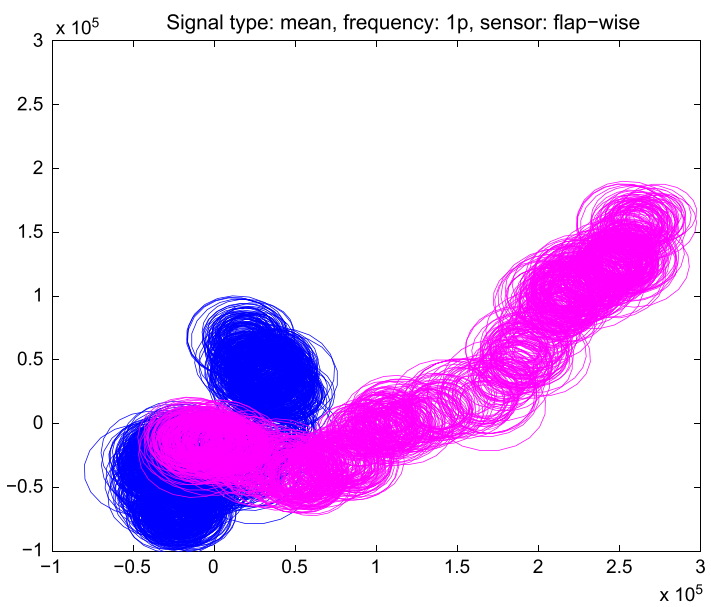

(A) $V_{0,1 p}$

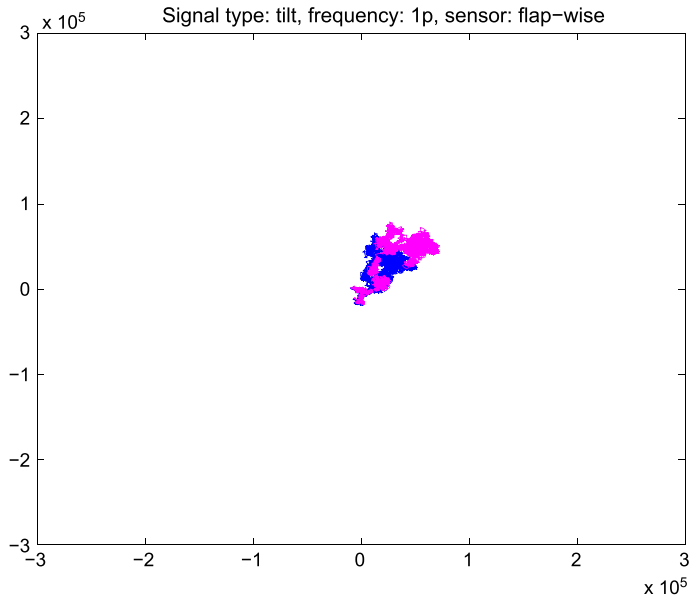

(B) $V_{c, 1 p}$

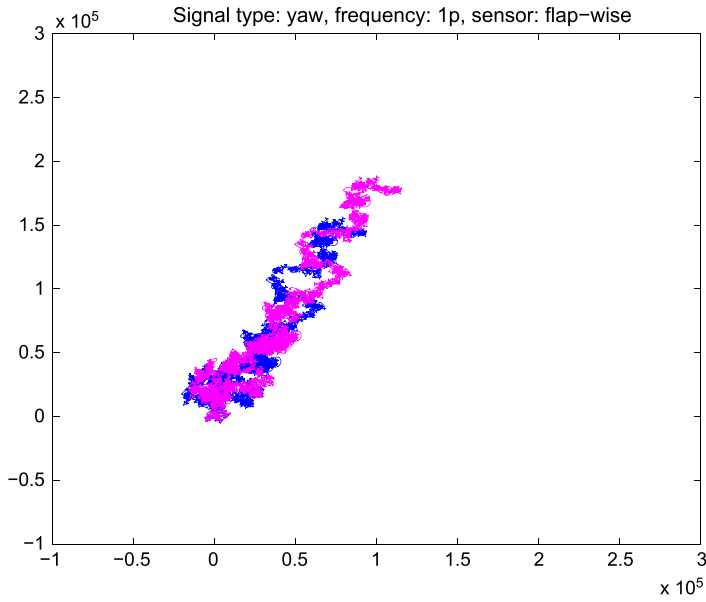

(C) $V_{s, 1 p}$

FIGURE 18 The integrated $1 p$ modulation signals for the flap-wise moments for mass imbalance on 1 blade. The blue curve is for the fault-free case [Colour figure can be viewed at wileyonlinelibrary.com]

faults can be clearly observed for the edge-wise signals. We obtain a significant indication of faults using the flap-wise signals. Furthermore, the $120^{\circ}$ phase difference between the different blades as indicated in the theory can be observed.

It is possible to isolate the different blade faults from each other. Furthermore, additive actuator faults can be isolated from mass imbalances and other types of faults, as described below.

\section{6 | DISCUSSION OF THE RESULTS}

Several issues related to the described method and simulation results will be discussed in the following section.

\section{1 | Fault isolation}

The simulations presented above consider only 2 types of faults: additive actuator faults and a mass imbalance on a blade. The above simulations illustrate that each fault results in different signatures in the modulation signals. Thus, it is possible to isolate these faults from each other. Furthermore, it is possible to isolate the blade to which the fault is related.

Other faults in the rotor system can also be isolated. However, the isolability of certain faults depends on the operation mode. This is the case for actuator faults. In the partial-load region, the wind turbine operates with a constant pitch angle. In this case, it is not possible to isolate additive actuator faults from multiplicative actuator faults, whereas this is possible in the full-load region. 


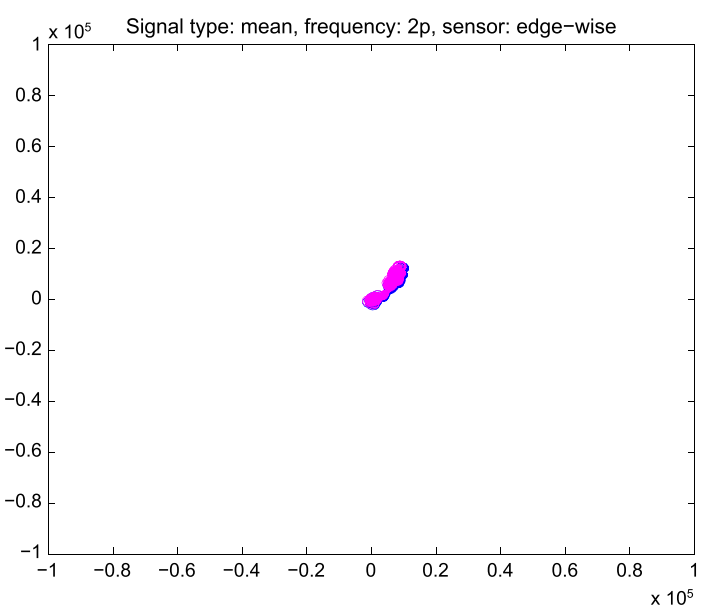

(A) $V_{0,2 p}$

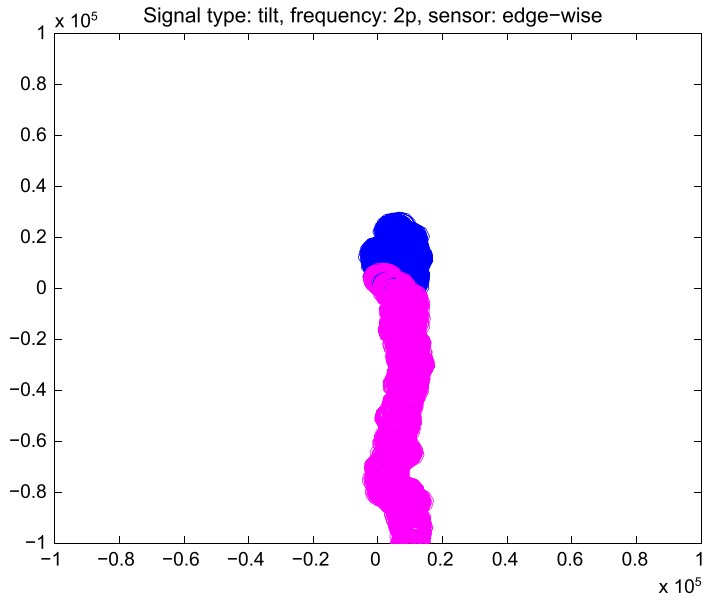

(B) $V_{c, 2 p}$

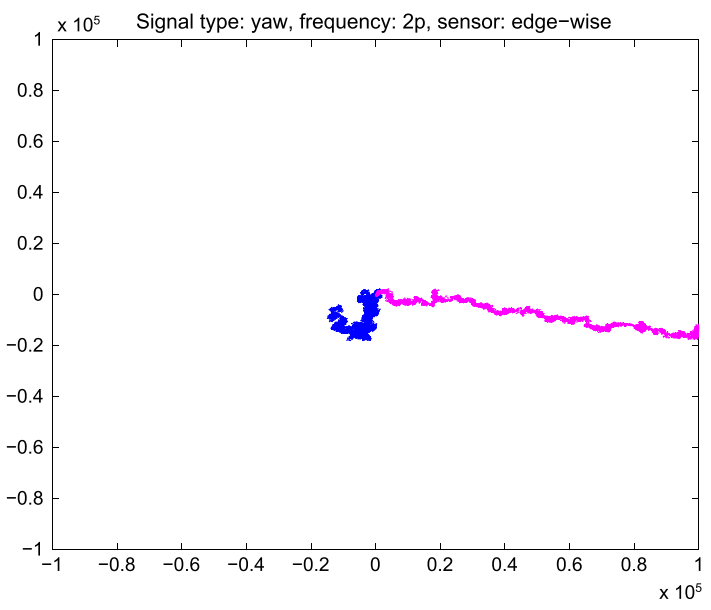

(C) $V_{s, 2 p}$

FIGURE 19 The integrated $2 p$ modulation signals for the edge-wise moments for mass imbalance on 1 blade. The blue curve is for the fault-free case [Colour figure can be viewed at wileyonlinelibrary.com]

The last type of faults are sensor faults, both additive and multiplicative. In the case where the root bending moments are not used for feedback control, as is typical for collective pitch control, isolation of sensor faults is reasonably easy. A fault in one set of sensors (flap-wise or edge-wise moment sensors) will not give a signature in modulation signals based on the other set of sensors. This is unique to faults in the sensors and allows sensor faults to be isolated from other faults in the rotor system. In the case where the root bending moments are used for feedback, there will be a minor coupling through the system, but as we have seen, a fault in one set of sensors will mainly result in signatures in the modulation signals based on the associated sensors.

A natural concern regarding individual pitch control is the extent to which the controller compensates for, eg, an actuator fault. The extent will depend on the objective of the controller. The IPC applied in the above simulations was designed to optimize the power production. An actuator fault on 1 blade results in a change in the pitch angles for all 3 blades to obtain the maximal power production. Thus, the fault diagnosis is not more difficult compared to the case where collective pitch control is applied. In some cases, it even results in a faster fault diagnosis because the modulation signals increase in amplitude.

The simulation results for only the 2 faults discussed above do provide a clear picture of the isolability of the different faults in the rotor system. Therefore, other simulations have been conducted under different operation conditions as well as with different types of faults. The simulations were conducted in both the full-load region and partial-load region, with both collective pitch control and individual pitch control. Sensor faults, actuator faults and blade faults were applied in the simulations. The results of these simulations are that each considered fault yields a unique signature in the modulation signals such that fault isolation is possible. Furthermore, without using the phase information from the modulation signals, it is still possible to isolate the different faults from each other but not isolate the blade to which the fault is related. 


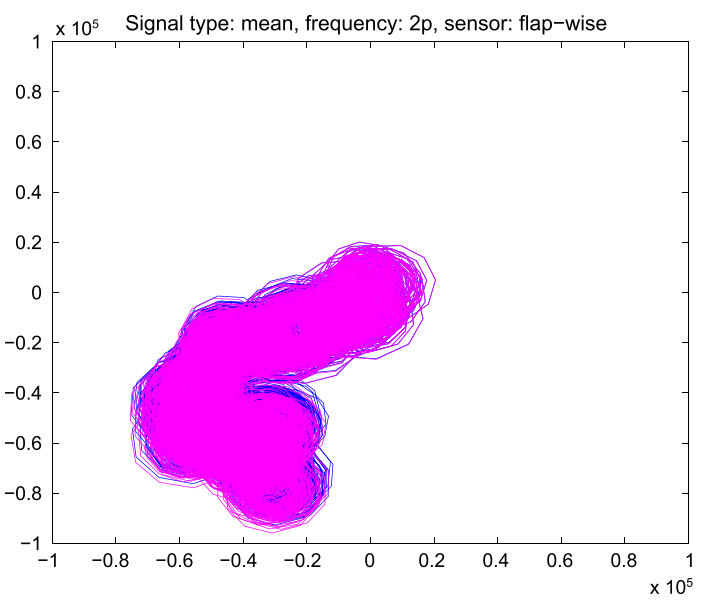

(A) $V_{0,2 p}$

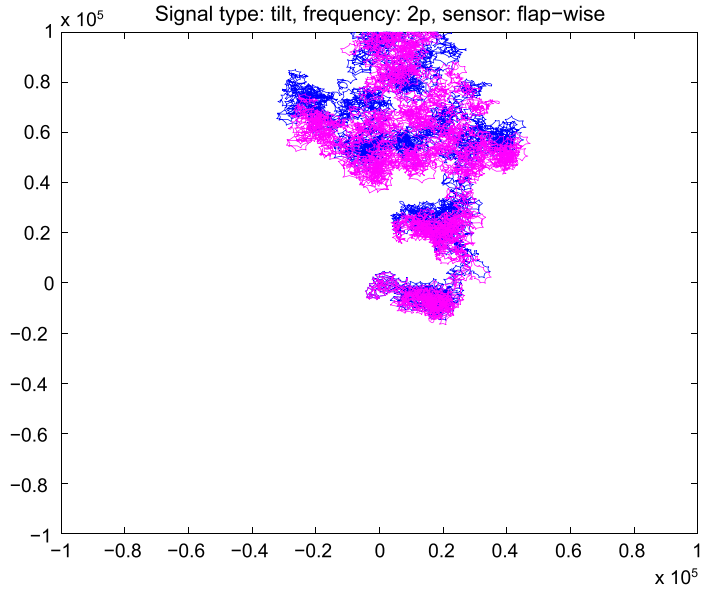

(B) $V_{c, 2 p}$

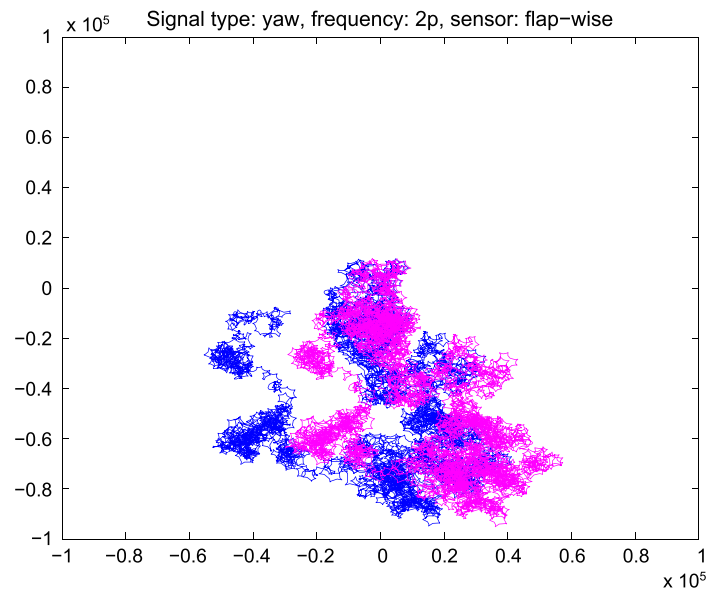

(C) $V_{s, 2 p}$

FIGURE 20 The integrated $2 p$ modulation signals for the flap-wise moments for mass imbalance on 1 blade. The blue curve is for the fault-free case [Colour figure can be viewed at wileyonlinelibrary.com]

Simulation results in the partial-load region using a collective pitch controller are shown in Table 3 . The different types of faults that were applied in the simulations are given in Table 4. Here, multiplicative actuator faults can describe, eg, a change in the actuator gain. Additive sensor faults could be an offset in the measurement, and a multiplicative sensor fault could be a change of the gain in the sensor. Please see Odgaard et $\mathrm{al}^{21}$ for additional details about possible faults.

The simulation results for the partial load region with different faults are shown in Table 3. An "X" indicates a large amplitude of the modulation signals, and an " $\mathrm{x}$ " indicates a small amplitude of the modulation signals. Further, an "I" indicates that the fault can be isolated.

First, it is possible to isolate all faults (indicated by an "I" in the last column in the table), except multiplicative actuator faults. This isolation is not possible because constant pitch angles are used in the partial load region; thus, it is impossible to isolate a multiplicative fault-it will be isolated as an additive actuator fault. For multiplicative sensor faults, the table also illustrates that they also have $1 p$ components in both $q_{c}^{f}$ and $q_{s}^{f}$ because of the offset in the root bending moment measurements, as described in Section 4.1.

Table 3 also illustrates that it is possible to isolate simultaneously occurring faults in the rotor system. Based on the different signatures, it is possible to isolate some simultaneously occurring faults, but this issue has not been investigated in detail.

\section{2 | Threshold selection}

An important aspect of fault detection and isolation is the selection of thresholds. A detailed analysis of the selection of thresholds has not been performed in this work. The simulations demonstrate that selection or design of thresholds is not simple. 


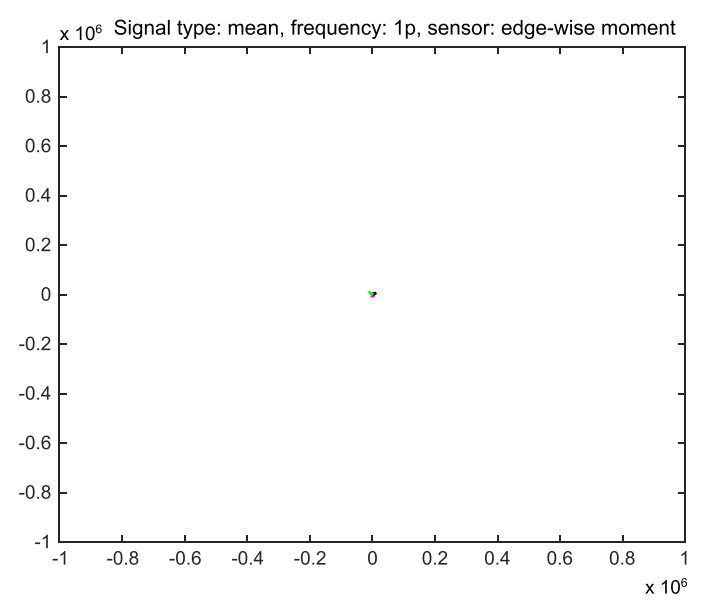

(A) $V_{0,1 p}$

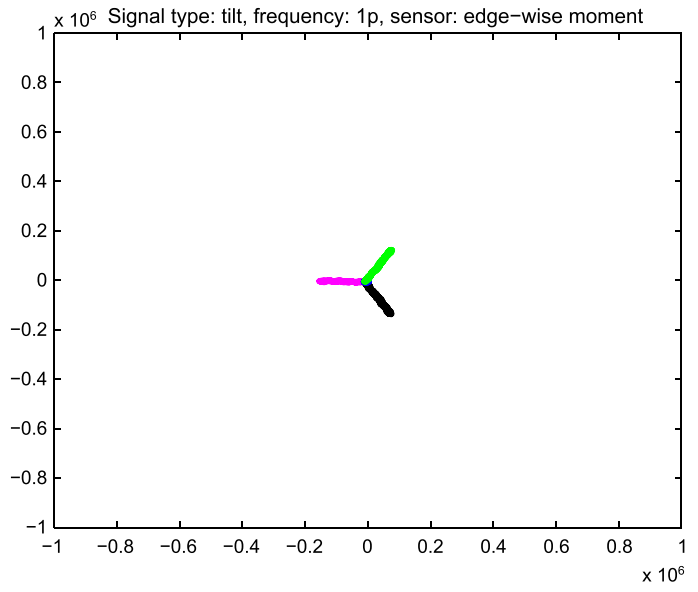

(B) $V_{c, 1 p}$

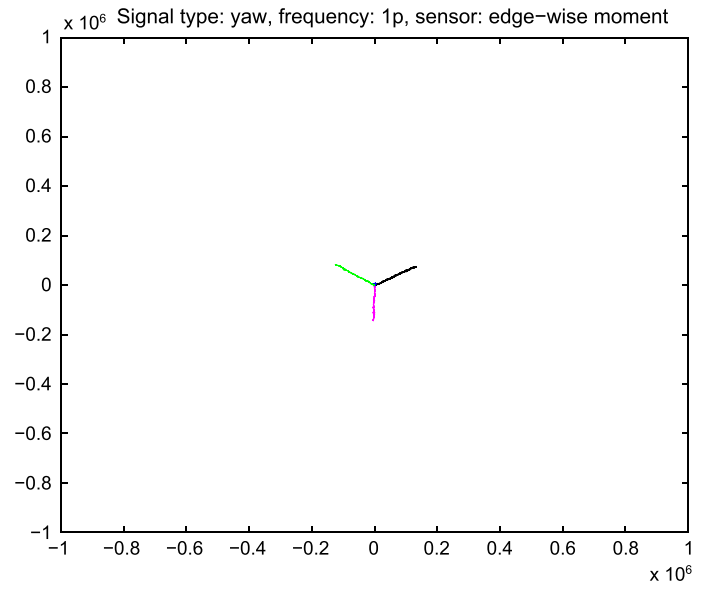

(C) $V_{s, 1 p}$

FIGURE 21 The integrated $1 p$ modulation signals for the edge-wise moments for additive actuator faults. The blue curve is for the fault-free case [Colour figure can be viewed at wileyonlinelibrary.com]

TABLE 3 Result of simulation in partial load with collective pitch control

\begin{tabular}{llllllllllllll} 
& $1 \boldsymbol{p}$ & $1 \boldsymbol{p}$ & $1 \boldsymbol{p}$ & $1 \boldsymbol{p}$ & $1 \boldsymbol{p}$ & $1 \boldsymbol{p}$ & $2 \boldsymbol{p}$ & $2 \boldsymbol{p}$ & $2 \boldsymbol{p}$ & $2 \boldsymbol{p}$ & $2 \boldsymbol{p}$ & $2 \boldsymbol{p}$ & \\
Fault & Edge & Flap & Edge & Flap & Edge & Flap & Edge & Flap & Edge & Flap & Edge & Flap & $q_{c}^{f}$ \\
Type & $q_{0}^{f}$ & $q_{0}^{f}$ & $q_{c}^{f}$ & $q_{c}^{f}$ & $q_{s}^{f}$ & $q_{s}^{f}$ & $q_{0}^{f}$ & $q_{0}^{f}$ & $q_{c}^{f}$ & $q_{c}^{f}$ & $q_{s}^{f}$ & $q_{s}^{f}$ & Isolation \\
\hline
\end{tabular}

Fault-free

Actuator add. $\begin{array}{llll}\text { X } & \mathrm{X} & \mathrm{X} & \mathrm{X}\end{array}$

$\mathrm{X}$

$\mathrm{x}$

$\mathrm{x}$

Acttuator mult.

Mass inbalance

Sensor, edge, add.

Sensor, edge, mult. $\quad \mathrm{X}$

Sensor, flap, add.

Sensor, flap, mult.

$\mathrm{x}-\mathrm{x}$

$\mathrm{x}$

$\mathrm{x}$

X

X

I

$\mathrm{x}$

$\begin{array}{ll}X & X \\ X & X\end{array}$

X

X

I

$\mathrm{X}$

I

An "X" indicates a large amplitude of the modulation signals, and an " $\mathrm{X}$ " indicates a small amplitude of the modulation signals. Further, an "I" indicates that the fault can be isolated.

Thresholds must be selected for each signal. This must be based on a calculation of the variation of the integrated modulation signals in the fault-free case. As shown by the simulations shown in Figures 19 to 22, the integrated modulation signals will not be zero in the fault-free case. After selecting a time window for the integration, it is possible to calculate the upper bounds for these variations. These bounds can be used as thresholds. 


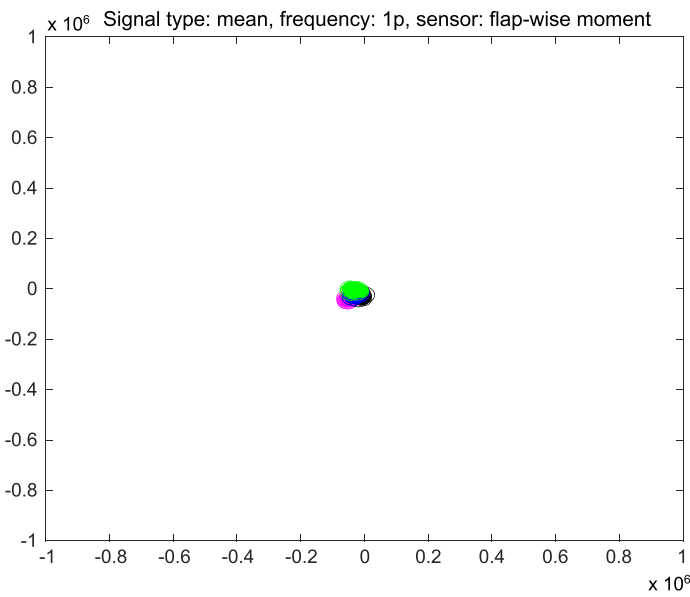

(A) $V_{0,1 p}$

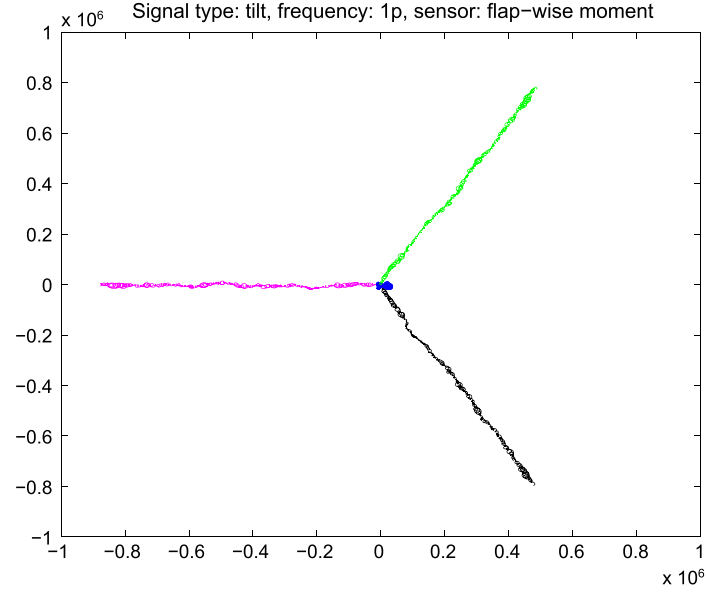

(B) $V_{c, 1 p}$

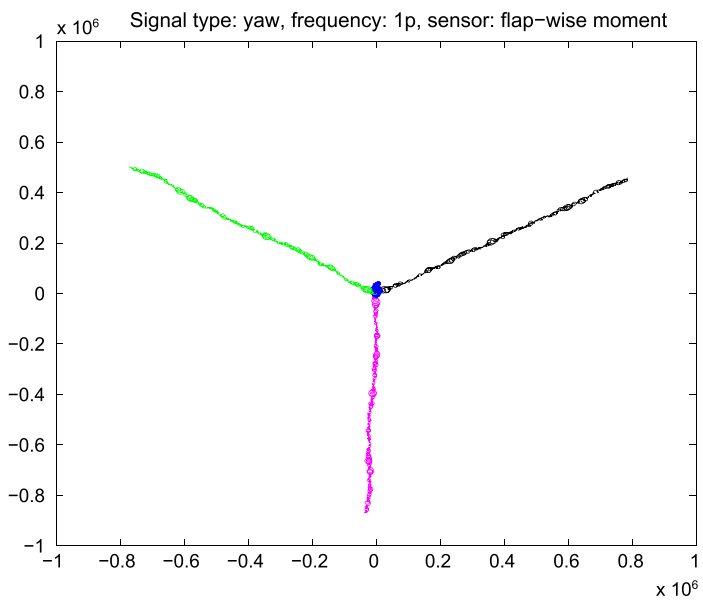

(C) $V_{s, 1 p}$

FIGURE 22 The integrated $1 p$ modulation signals for the edge-wise moments for additive actuator faults. The blue curve is for the fault-free case [Colour figure can be viewed at wileyonlinelibrary.com]

TABLE 4 Fault description

\begin{tabular}{ll} 
Fault Types & \multicolumn{1}{c}{ Fault Value } \\
\hline Additive fault on blade pitch actuator & 1 degree \\
\hline Multiplicative fault on blade pitch actuator & $10 \%$ \\
\hline Additive fault on edge-wise blade root bending moment sensors & $3 \%$ (of the maximal value) \\
\hline Additive fault on flap-wise blade root bending moment sensors & $3 \%$ (of the mean value) \\
\hline Multiplicative fault on blade root bending moment sensors & $10 \%$ \\
\hline Mass imbalance & $2 \%$ \\
\hline
\end{tabular}

\section{3 | Fault estimation and monitoring}

This paper described fault detection and isolation. However, it is possible to indirectly estimate a fault by the described method. Tables B1, B2, and B3 in Appendix B illustrate that the modulation signals are proportional to the faulty parameter $\delta$. The faulty parameter $\delta$ can typically only be estimated indirectly because the other gains included in the modulation will be unknown, as $q_{o f f}$ and $q_{s y m}$ are in Equations 19 and 21. Using a time window for the integration of the modulation signals, the increasing rate of the integrated signals is an indirect measure of the fault signal that occurred in the system.

Similarly, an estimation of the increasing rate for specific faults or changes in the rotor system can also be applied to monitor the system. If a parameter or fault in the rotor system increases over time, the amplitude of the associated modulation vectors 
will also increase over time. This will result in increasing rates of the integrated modulation signals, which provides a reasonably simple way to monitor changes in the rotor system while also being able to follow these changes over time.

\section{7 | CONCLUDING REMARKS}

This paper describes a model-free method* for the fault diagnosis and monitoring of rotor systems in wind turbines.

Faults that result in a change in the symmetry of wind turbine rotor systems can be detected and isolated without using a model. Using an MBC transformation of the blade moments followed by signal modulation yields a unique set of signals that can be directly applied to fault diagnosis in rotor systems. One of the key elements in this diagnosis is the application of the phase shift in the modulation vectors, which results in unique signatures in the modulation vector for each fault: blade faults, sensor faults, or actuator faults on each blade. This also includes the type of faults, either additive faults or multiplicative faults.

The modulation vectors can also be directly applied to the monitoring of variations in actuators, blades, and sensors. The length of these vectors is directly related to the change in the rotor system. Using periodic integration of these modulation vectors will allow for the monitoring of different parts of the rotor system over time.

The results in this paper provide a solid basis for further research in this area. The simulations yield promising results. The next step is to apply the developed diagnosis method to real data from wind turbines. Further, the aspect of simultaneously occurring faults will also be considered in more detail both in simulations as well as on real data from wind turbines.

\section{REFERENCES}

1. Blanke M, Kinnart M, Lunze J, Staroswiecki M. Diagnosis and Fault-Tolerant Control. Berlin Heidelberg: Springer, 2006.

2. Chen J, Patton R. Robust Model-Based Fault Diagnosis for Dynamic Systems. Boston: Kluwer Academic Publishers, 1998.

3. Gertler J. Fault Detection and Diagnosis in Engineering Systems. New York: Marcel Dekker, 1998.

4. Basseville M, Nikiforov IV. Detection of Abrupt Changes-Theory and Application. Englewood Cliffs: Prentice Hall, 1993.

5. Gustafsson F. Adaptive Filtering and Change Detection. Wiley \& Sons: Chichester, England, 2000.

6. Qin SJ. Survey on data-driven industrial process monitoring and diagnosis. Annu Rev Control 2012; 36(2):220-234.

7. Hameed Z, Hong Y, Cho Y, Ahn S, Song C. Condition monitoring and fault detection of wind turbines and related algorithms: a review. Renew Sustainable Energy Rev 2009; 13(1):1-39.

8. Marquez F, Tobias AM, Perez J, Papaelias M. Condition monitoring of wind turbines: techniques and methods. Renew Energy 2012; 46: 169-178.

9. Tchakoua P, Wamkeue R, Ouhrouche M, Slaoui-Hasnaoui F, Tameghe T, Ekemb G. Wind turbine condition monitoring: state-of-the-art review, new trends, and future challenges. Energies 2014; 7: 2595-2630.

10. Sheng S, Veers P Wind turbine drivetrain condition monitoring—an overview. Mechanical Failures Prevention Group: Applied Systems Health Management Conference, Virginia Beach, Virginia, USA, 2011.

11. Wei X, Verhaegen M, van Engelen T. Sensor fault detection and isolation for wind turbines based on subspace identification and Kalman filter techniques. Int J Adapt Control Signal Proces 2010; 24(8):687-707.

12. Wei X, Verhaegen M. Sensor and actuator fault diagnosis for wind turbine systems by using robust observer and filter. Wind Energy 2011; 14(4):491-516.

13. Saad C, Mostafa B, Abderrahmane H. Performance analysis of faults detection in wind turbine generator based on high-resolution frequency estimation methods. Int J Adv Comput Sci Appl 2014; 3(4):139-148.

14. Watson S, Xiang B, Yang W, Tavner P, Crabtree C. Condition monitoring of the power output of wind turbine generators using wavelets. IEEE Trans Energy Convers 2010; 25(3):715-721.

15. Gong X, Qiao W. Imbalance fault detection of direct-drive wind turbines using generator current signals. IEEE Trans Energy Convers 2012; 27(2):468-476.

16. Feng Z, Liang M, Zhang Y, Hou S. Fault diagnosis for wind turbine planetary gearboxes via demodulation analysis based on ensemble empirical mode decomposition and energy separation. Renew 2012; 47: 112-126.

17. Amirat $\mathrm{Y}$, Choqueuse V, Benbouzid M Wind turbines condition monitoring and fault diagnosis using generator current amplitude demodulation. IEEE International Energy Conference, Manama, Bahrain; 2010; 310-315.

18. Qiao W. Recovery act: online nonintrusive condition monitoring and fault detection for wind turbines. Technical Report, Department of Electrical Engineering, University of Nebraska - Lincoln, USA, 2012.

19. Hang J, Zhang J, Cheng M. Fault diagnosis of wind turbine based on multisensors information fusion technology. IET Renew Power Gener 2013; 8(3):289-298.

20. Kusiak A, Verma A. A data-driven approach for monitoring blade pitch faults in wind turbines. IEEE Trans Sustainable Energy 2011; 2(1):87-96.

21. Odgaard PF, Stoustrup J, Kinnaert M. Fault-tolerant control of wind turbines: a benchmark model. IEEE Trans Control Syst Technol 2013; 21(4):1168-1182.

*A patent application including the described method has been submitted. 
22. Henriksen L, Niemann H, Poulsen N Detecting asymmetries in the rotor of a wind turbine using the multi-blade coordinate transformation. Diagnostics of Processes and Systems, Zamosc, Poland, 2011:57-67.

23. Henriksen L, Niemann H, Poulsen N Fault diagnosis of a wind turbine rotor using a multi-blade coordinate framework. Preprints of the 8th IFAC Symposium on Fault Detection, Supervision and Safety of Technical Processes (SAFEPROCESS), Mexico City, Mexico, 2012; 37-42.

24. Henriksen L, Niemann H, Poulsen N Monitoring of a wind turbine rotor using a multi-blade coordinate framework. Preprints of the 16th IFAC Symposium on System Identification, the International Federation of Automatic Control, Brussels, Belgium, 2012; 374-379.

25. Bir G Multiblade coordinate transformation and its application to wind turbine analysis. ASME Wind Symposium, Reno, Nevada, USA, 2008.

26. Jonkman J, Butterfield S, Musial W, Scott G. Definition of a 5MW reference wind turbine for offshore system development. Technical Rport, National Renewable Energy Laboratory, 1617 Cole Boulevard, Golden, Colorado 80401-3393 303-275-3000, 2009.

27. Stol K, Moll H, Bir G, Namik H A comparison of multi-blade coordinate transformation and direct periodic techniques for wind turbine control design. 47th AIAA Aerospace Sciences Meeting Including the new Horizons Forum and Aerospace Exposition, Orlando, Florida, USA, 2009.

28. Jonkman JM, Marshall LB Jr.Fast user's guide. Technical Report NREL/EL-500-38230, National Renewable Energy Laboratory, Golden, CO, 2005.

29. Bossanyi E. Individual blade pitch control for load reduction. Wind Energy 2003; 6: 119-128.

30. Niemann H, Mirzaei M, Henriksen L, Poulsen N Diagnosis of wind turbine rotor system. Proceedings of the American Control Conference, Boston, MA, USA, 2016; 3170-3175.

How to cite this article: Niemann H, Kjoelstad Poulsen N, Mirzaei M, Henriksen LC. Fault diagnosis and condition monitoring of Wind turbines. Int J Adapt Control Signal Process. 2017;0:1-28. https://doi.org/10.1002/acs.2782

\section{APPENDIX A A: PHASE PERTURBATION}

Let a phase perturbation $q$ be given by

$$
\begin{aligned}
q & =\left[\begin{array}{c}
\sin \left(\phi_{1}(t)-\phi_{0}\right) \\
\sin \left(\phi_{2}(t)-\phi_{0}\right) \\
\sin \left(\phi_{3}(t)-\phi_{0}-\phi_{f, 3}\right)
\end{array}\right] \\
& =\left[\begin{array}{c}
\sin \left(\phi_{1}(t)-\phi_{0}\right) \\
\sin \left(\phi_{2}(t)-\phi_{0}\right) \\
\sin \left(\phi_{3}(t)-\phi_{0}\right)+\left(\sin \left(\phi_{3}(t)-\phi_{0}-\phi_{f, 3}\right)-\sin \left(\phi_{3}(t)-\phi_{0}\right)\right)
\end{array}\right] \\
& =\left[\begin{array}{c}
\sin (\Omega t) \\
\sin \left(\Omega t-\frac{2}{3} \pi\right) \\
\sin \left(\Omega t-\frac{4}{3} \pi\right)-2 \cos \left(\Omega t-\frac{4}{3} \pi-\frac{1}{2} \phi_{f, 3}\right) \sin \left(\frac{1}{2} \phi_{f, 3}\right)
\end{array}\right],
\end{aligned}
$$

ie, a perturbation at $q_{3}$. In the fixed frame, $q^{f}$ is given by

$$
\begin{aligned}
q^{f} & =\left[\begin{array}{c}
0 \\
-\sin \left(\phi_{0}\right) \\
\cos \left(\phi_{0}\right)
\end{array}\right] \\
& +\left[\begin{array}{c}
-\frac{2}{3} \sin \left(\frac{1}{2} \phi_{f, 3}\right) \cos \left(\Omega t-\frac{4}{3} \pi-\frac{1}{2} \phi_{f, 3}\right) \\
-\frac{2}{3} \sin \left(\frac{1}{2} \phi_{f, 3}\right) \cos \left(\phi_{0} i+\frac{1}{2} \phi_{f, 3}\right)-\frac{2}{3} \sin \left(\frac{1}{2} \phi_{f, 3}\right) \cos \left(2 \Omega t+\phi_{0}-\frac{2}{3} \pi-\frac{1}{2} \phi_{f, 3}\right) \\
-\frac{2}{3} \sin \left(\frac{1}{2} \phi_{f, 3}\right) \sin \left(\phi_{0}+\frac{1}{2} \phi_{f, 3}\right)-\frac{2}{3} \sin \left(\frac{1}{2} \phi_{f, 3}\right) \sin \left(2 \Omega t+\phi_{0}-\frac{2}{3} \pi-\frac{1}{2} \phi_{f, 3}\right)
\end{array}\right] \\
& =\left[\begin{array}{c}
0 \\
-\sin \left(\phi_{0}\right)-\frac{2}{3} \sin \left(\frac{1}{2} \phi_{f, 3}\right) \cos \left(\phi_{0}+\frac{1}{2} \phi_{f, 3}\right) \\
\cos \left(\phi_{0}\right)-\frac{2}{3} \sin \left(\frac{1}{2} \phi_{f, 3}\right) \sin \left(\phi_{0}+\frac{1}{2} \phi_{f, 3}\right)
\end{array}\right] \\
& +\left[\begin{array}{c}
-\frac{2}{3} \sin \left(\frac{1}{2} \phi_{f, 3}\right) \cos \left(\Omega t-\frac{4}{3} \pi-\frac{1}{2} \phi_{f, 3}\right) \\
-\frac{2}{3} \sin \left(\frac{1}{2} \phi_{f, 3}\right) \cos \left(2 \Omega t+\phi_{0}-\frac{2}{3} \pi-\frac{1}{2} \phi_{f, 3}\right) \\
-\frac{2}{3} \sin \left(\frac{1}{2} \phi_{f, 3}\right) \sin \left(2 \Omega t+\phi_{0}-\frac{2}{3} \pi-\frac{1}{2} \phi_{f, 3}\right)
\end{array}\right] .
\end{aligned}
$$


Equation A2 illustrates that a phase perturbation gives a $1 p$-variation of $\boldsymbol{q}_{0}$ and a $2 p$-variation in $\boldsymbol{q}_{c}$ and $\boldsymbol{q}_{s}$, equivalent to the multiplicative perturbations.

In the phase case, all 3 terms in $q^{f}$ result in a $1 p$ variation or $2 p$ variation. From Equation A2, the 3 terms related to the phase faults in input no. 3 are given by

$$
\left[\begin{array}{c}
q_{0}^{f} \\
q_{c}^{f} \\
q_{s}^{f}
\end{array}\right]=-\frac{2}{3} \sin \left(\frac{1}{2} \phi_{f, 3}\right)\left[\begin{array}{c}
\cos \left(\Omega t-\frac{4}{3} \pi-\frac{1}{2} \phi_{f, 3}\right) \\
\cos \left(2 \Omega t+\phi_{0}-\frac{2}{3} \pi-\frac{1}{2} \phi_{f, 3}\right) \\
\sin \left(2 \Omega t+\phi_{0}-\frac{2}{3} \pi-\frac{1}{2} \phi_{f, 3}\right)
\end{array}\right] .
$$

Following the same process as for the multiplicative faults, the first term in Equation A3 is multiplied by $\cos (\Omega t)$ and $\sin (\Omega t)$ and the last 2 terms are multiplied by $\cos (2 \Omega t)$ and $\sin (2 \Omega t)$. This yields the modulation vectors shown in Table B3.

It is again possible to isolate which input (blade) the fault is related to based on the above results. The modulation vectors differ from the modulation vectors for multiplicative faults.

\section{APPENDIX B B: MODULATION VECTORS}

Tables B1, B2, and B3 present the modulation vectors for additive faults, multiplicative faults, and phase faults, respectively.

TABLE B1 Modulation vectors for additive faults

Modulation vectors for additive faults

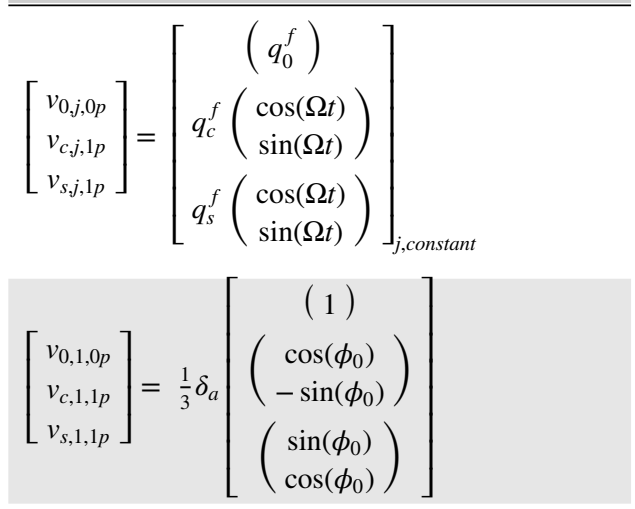

$$
\left[\begin{array}{c}
v_{0,2,0 p} \\
v_{c, 2,1 p} \\
v_{s, 2,1 p}
\end{array}\right]=\frac{1}{3} \delta_{a}\left[\begin{array}{c}
(1) \\
\left(\begin{array}{c}
\cos \left(-\frac{2}{3} \pi+\phi_{0}\right) \\
-\sin \left(-\frac{2}{3} \pi+\phi_{0}\right)
\end{array}\right) \\
\left(\begin{array}{c}
\sin \left(-\frac{2}{3} \pi+\phi_{0}\right) \\
\cos \left(-\frac{2}{3} \pi+\phi_{0}\right)
\end{array}\right)
\end{array}\right]
$$

$$
\left[\begin{array}{c}
v_{0,3,0} \\
v_{c, 3,1 p} \\
v_{s, 3,1 p}
\end{array}\right]=\frac{1}{3} \delta_{a}\left[\begin{array}{c}
(1) \\
\cos \left(-\frac{4}{3} \pi+\phi_{0}\right) \\
-\sin \left(-\frac{4}{3} \pi+\phi_{0}\right)
\end{array}\right)
$$


TABLE B2 Modulation vectors for multiplicative faults

Modulation vectors for multiplicative faults

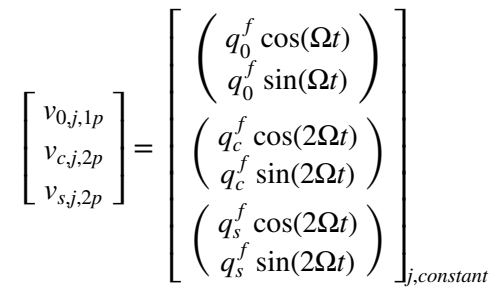$$
\left[\begin{array}{c}
v_{0,1,1 p} \\
v_{c, 1,2 p} \\
v_{s, 1,2 p}
\end{array}\right]=\frac{1}{6} \delta_{m}\left[\begin{array}{c}
\left(\begin{array}{c}
0 \\
1
\end{array}\right) \\
\left(\begin{array}{c}
\sin \left(\phi_{0}\right) \\
\cos \left(\phi_{0}\right)
\end{array}\right) \\
\left(\begin{array}{c}
\cos \left(\phi_{0}\right) \\
-\sin \left(\phi_{0}\right)
\end{array}\right)
\end{array}\right]
$$

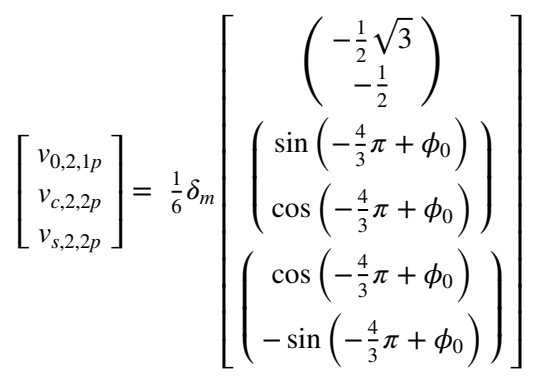

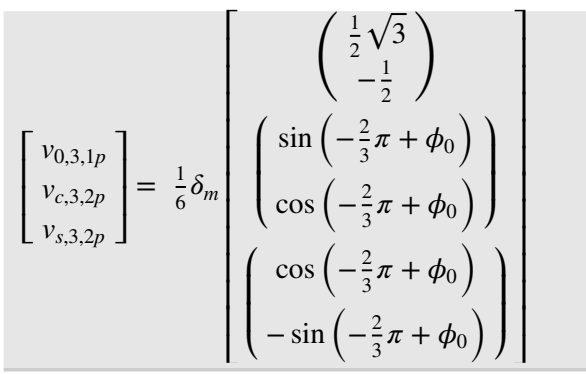


28

WILEY

NIEMANN ET AL.

TABLE B3 Modulation vectors for phase faults

Modulation vectors for phase faults

$$
\begin{aligned}
& {\left[\begin{array}{c}
v_{0, j, 1 p} \\
v_{c, j, 2 p} \\
v_{s, j, 2 p}
\end{array}\right]=\left[\begin{array}{c}
\left(\begin{array}{c}
q_{0}^{f} \cos (\Omega t) \\
q_{0}^{f} \sin (\Omega t)
\end{array}\right) \\
\left(\begin{array}{c}
q_{c}^{f} \cos (2 \Omega t) \\
q_{c}^{f} \sin (2 \Omega t)
\end{array}\right) \\
\left(\begin{array}{c}
q_{s}^{f} \cos (2 \Omega t) \\
q_{s}^{f} \sin (2 \Omega t)
\end{array}\right)
\end{array}\right]_{j, \text { constant }}} \\
& {\left[\begin{array}{c}
v_{0,1,1 p} \\
v_{c, 1,2 p} \\
v_{s, 1,2 p}
\end{array}\right]=\frac{1}{3} \sin \left(\frac{1}{2} \phi_{f, 1}\right)\left[\begin{array}{c}
\left(\begin{array}{c}
-\cos \left(-\frac{1}{2} \phi_{f, 3}\right) \\
\sin \left(-\frac{1}{2} \phi_{f, 3}\right)
\end{array}\right) \\
\left(\begin{array}{c}
-\cos \left(\phi_{0}-\frac{1}{2} \phi_{f, 3}\right) \\
\sin \left(\phi_{0}-\frac{1}{2} \phi_{f, 3}\right)
\end{array}\right) \\
\left(\begin{array}{c}
-\sin \left(\phi_{0}-\frac{1}{2} \phi_{f, 3}\right) \\
-\cos \left(\phi_{0}-\frac{1}{2} \phi_{f, 3}\right)
\end{array}\right)
\end{array}\right]} \\
& {\left[\begin{array}{l}
v_{0,2,1 p} \\
v_{c, 2,2 p} \\
v_{s, 2,2 p}
\end{array}\right]=\frac{1}{3} \sin \left(\frac{1}{2} \phi_{f, 2}\right)\left[\begin{array}{c}
\left(\begin{array}{c}
-\cos \left(-\frac{2}{3} \pi-\frac{1}{2} \phi_{f, 3}\right) \\
\sin \left(-\frac{2}{3} \pi-\frac{1}{2} \phi_{f, 3}\right)
\end{array}\right) \\
\left(\begin{array}{c}
-\cos \left(-\frac{4}{3} \pi+\phi_{0}-\frac{1}{2} \phi_{f, 3}\right) \\
\sin \left(-\frac{4}{3} \pi+\phi_{0}-\frac{1}{2} \phi_{f, 3}\right)
\end{array}\right) \\
\left(\begin{array}{c}
-\sin \left(-\frac{4}{3} \pi+\phi_{0}-\frac{1}{2} \phi_{f, 3}\right) \\
-\cos \left(-\frac{4}{3} \pi+\phi_{0}-\frac{1}{2} \phi_{f, 3}\right)
\end{array}\right)
\end{array}\right]} \\
& {\left[\begin{array}{c}
v_{0,3,1 p} \\
v_{c, 3,2 p} \\
v_{s, 3,2 p}
\end{array}\right]=\frac{1}{3} \sin \left(\frac{1}{2} \phi_{f, 3}\right)\left[\begin{array}{c}
\left(\begin{array}{c}
-\cos \left(-\frac{4}{3} \pi-\frac{1}{2} \phi_{f, 3}\right) \\
\sin \left(-\frac{4}{3} \pi-\frac{1}{2} \phi_{f, 3}\right)
\end{array}\right) \\
\left(\begin{array}{c}
-\cos \left(-\frac{2}{3} \pi+\phi_{0}-\frac{1}{2} \phi_{f, 3}\right) \\
\sin \left(-\frac{2}{3} \pi+\phi_{0}-\frac{1}{2} \phi_{f, 3}\right)
\end{array}\right) \\
\left(\begin{array}{c}
-\sin \left(-\frac{2}{3} \pi+\phi_{0}-\frac{1}{2} \phi_{f, 3}\right. \\
-\cos \left(-\frac{2}{3} \pi+\phi_{0}-\frac{1}{2} \phi_{f, 3}\right)
\end{array}\right)
\end{array}\right]}
\end{aligned}
$$

